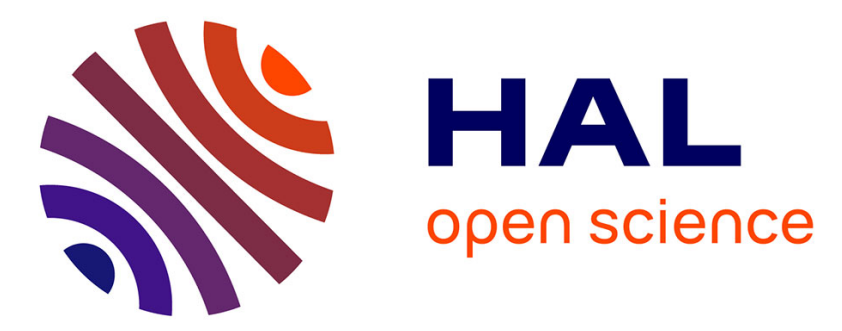

\title{
Analysis of a system modelling the motion of a piston in a viscous gas
}

Debayan Maity, Takéo Takahashi, Marius Tucsnak

\section{To cite this version:}

Debayan Maity, Takéo Takahashi, Marius Tucsnak. Analysis of a system modelling the motion of a piston in a viscous gas. Journal of Mathematical Fluid Mechanics, 2017, 19 (3), pp.551-579. 10.1007/s00021-016-0293-2 . hal-01285089

\section{HAL Id: hal-01285089 \\ https://hal.science/hal-01285089}

Submitted on 8 Mar 2016

HAL is a multi-disciplinary open access archive for the deposit and dissemination of scientific research documents, whether they are published or not. The documents may come from teaching and research institutions in France or abroad, or from public or private research centers.
L'archive ouverte pluridisciplinaire HAL, est destinée au dépôt et à la diffusion de documents scientifiques de niveau recherche, publiés ou non, émanant des établissements d'enseignement et de recherche français ou étrangers, des laboratoires publics ou privés. 


\title{
ANALYSIS OF A SYSTEM MODELLING THE MOTION OF A PISTON IN A VISCOUS GAS
}

\author{
DEBAYAN MAITY, TAKÉO TAKAHASHI, AND MARIUS TUCSNAK
}

\begin{abstract}
We study a free boundary problem modelling the motion of a piston in a viscous gas. The gas-piston system fills a cylinder with fixed extremities, which possibly allow gas from the exterior to penetrate inside the cylinder. The gas is modeled by the 1D compressible Navier-Stokes system and the piston motion is described by the second Newton's law. We prove the existence and uniqueness of global in time strong solutions. The main novelty brought in by our results is that they include the case on nonhomogeneous boundary conditions which, as far as we know, have not been studied in this context. Moreover, even for homogeneous boundary conditions, our results require less regularity of the initial data than those obtained in previous works.
\end{abstract}

Key words. Compressible Navier-Stokes System, fluid-particle interaction, strong solutions. AMS subject classifications. 35Q30, 76D05, 76N10

\section{Contents}

1. Introduction and main results

2. Change of variables and definition of strong solutions

3. Local in Time Existence and uniqueness

4. Mass and energy estimates

5. Estimates on the density field

6. Proof of Theorem 1.1

Appendix A. Some background on the linear transport equation 25

References

\section{INTRODUCTION AND MAIN RESULTS}

We consider a one dimensional model for the motion of a particle (piston) in a cylinder filled with a viscous compressible gas. The extremities of the cylinder are fixed, but the gas is allowed to penetrate inside the cylinder. The gas is modelled by the 1D compressible Navier-Stokes equations, whereas the piston obeys Newton's second law. Since the position of the piston (and, consequently, the domain occupied by the gas) is one of the unknowns of the problem, we have a free boundary value problem. Our initial motivation was of control theoretic nature: we aimed proving that we can steer the gas to rest and the piston to any final position by injecting gas at the extremities of the cylinder. We intended in this way to transpose to this physically motivated model our previous results obtained for a toy model, in which the compressible Navier-Stokes equations are replaced by the viscous Burgers equation. We refer to Vázquez and Zuazua [21, 22] for the description and the analysis of the toy model and Liu et al. [13] and Cîndea et al [6] for the associated control problems. We quickly realized that the major difficulty to be solved in order to accomplish the proposed goal consists in proving the global in time existence and uniqueness of solutions, in appropriate function spaces. Indeed, to our knowledge, the free boundary problem we consider has not been tackled in the literature in the case of a non vanishing gas velocity at the extremities of the cylinder (i.e., with non homogeneous boundary conditions). The main difficulties are induced by the simultaneous presence of the the two fixed ends where the fluid can penetrate inside the cylinder

Date: March 8, 2016. 
(easier to describe in an Eulerian setting) and of the free moving impermeable piston, which is easier studied in mass Lagrangian coordinates.

In the case of homogeneous boundary conditions for the velocity, the piston problem has been tackled by Shelukhin [18], [17], using functional spaces more regular than the ones which appear below (see also Shelukhin [19], where the viscous gas and the piston are supposed to be heat conducting). The problem has been revisited (still with homogeneous boundary conditions) in Antman and Wilber [1], where the emphasis is the treatment of the asymptotic behavior of solutions as the ratio of the mass of the gas and of the mass of the piston tends to zero. Tackling non homogeneous boundary conditions is a delicate question even in the case in which the gas evolves alone in a fixed cylinder, i.e., if there is no piston inside (see the comments at the beginning of Section 2 below).

Another class of references related to our work tackles the analysis of the equations modeling the evolution of a viscous gas-rigid body system which fills an open set of $\mathbb{R}^{3}$. In this case, as far as we know, the existing results concern homogeneous boundary conditions for the velocity and they assert either the existence and uniqueness of smooth solutions (global in time for small initial data in Boulakia and Guerrero [4] and local in time in Hieber and Murata [11]) or the existence of weak solutions, see Desjardins and Esteban [7] and Feireisl [10]. Let us also mention that recently the problem of steering a viscous gas, without considering a moving piston, to a given final state in a finite time (the controllability problem) received an increasing attention (see, for instance, Ervedoza et al [9, 8], Maity [14] and references therein).

The system we study in this work is described by the equations

$$
\begin{array}{lr}
\partial_{t} \rho+\partial_{x}(\rho u)=0 & (t \geqslant 0, x \in[-1,1] \backslash\{h(t)\}), \\
\rho\left(\partial_{t} u+u \partial_{x} u\right)-\partial_{x x} u+\partial_{x}\left(\rho^{\gamma}\right)=0 & (t \geqslant 0, x \in[-1,1] \backslash\{h(t)\}), \\
u(t, h(t))=\dot{h}(t) & (t \geqslant 0), \\
m \ddot{h}(t)=\left[\partial_{x} u-\rho^{\gamma}\right](t, h(t)) & (t \geqslant 0),
\end{array}
$$

with the initial conditions

$$
\left\{\begin{array}{l}
h(0)=h_{0}, \quad \dot{h}(0)=\ell_{0}, \\
u(0, x)=u_{0}(x), \quad \rho(0, x)=\rho_{0}(x) \quad\left(x \in[-1,1] \backslash\left\{h_{0}\right\}\right) .
\end{array}\right.
$$

In the above equations $\gamma \geqslant 1$ is a constant, $\rho$ stands for the density field, $u$ denotes the velocity field of the fluid (both in Eulerian coordinates), $m$ is the mass of the particle and $h(t)$ is, for every $t \geqslant 0$, its position at instant $t$. If $f$ is a real function of $t \geqslant 0$ and of $x \in \mathbb{R}$, the symbol $[f](t, x)$ stands for the jump at instant $t$ of $f$ at $x$, i.e.,

$$
[f](t, x)=f\left(t, x^{+}\right)-f\left(t, x^{-}\right) .
$$

The notation $\dot{h}$ and $\ddot{h}$ corresponds to the first and the second derivatives of $h$. We will use this last notation for functions depending only on time.

Our main result concerns the existence and uniqueness of global in time strong solutions of the initial and boundary value problem (1.1), (1.2) with possibly non homogeneous boundary conditions. To state our main result we complete (1.1), (1.2) by one of the sets of boundary conditions

$$
\begin{array}{cc} 
\begin{cases}u(t,-1)=u_{-1}(t)>0, \quad u(t, 1)=0 & (t \geqslant 0), \\
\rho(t,-1)=\rho_{-1}(t) & (t \geqslant 0),\end{cases} \\
u(t,-1)=0, \quad u(t, 1)=0 & (t \geqslant 0),
\end{array}
$$

or

$$
\begin{cases}u(t,-1)=u_{-1}(t), \quad u(t, 1)=-u_{1}(t) & (t \geqslant 0), \\ u_{-1}(t)>0, \quad u_{1}(t)>0 & (t \geqslant 0), \\ \rho(t,-1)=\rho_{-1}(t) & (t \geqslant 0), \\ \rho(t, 1)=\rho_{1}(t) & (t \geqslant 0) .\end{cases}
$$

Theorem 1.1. Let $T>0$ and assume that $h_{0} \in(-1,1), \ell_{0} \in \mathbb{R}$ and that the functions $u_{0}$ in $(1.2)$ and $u_{-1}, u_{1}$, $\rho_{-1}$ and $\rho_{1}$ in (1.5) satisfy the conditions: 
(H1) $u_{0} \in H^{1}(-1,1)$ and $u_{0}\left(h_{0}\right)=\ell_{0}$;

(H2) $\rho_{0} \in H^{1}\left(-1, h_{0}\right) \cap H^{1}\left(h_{0}, 1\right)$ and $\rho_{0}(x)>0$ for every $x \in[-1,1] \backslash\left\{h_{0}\right\}$.

(H3) $u_{-1}, u_{1} \in H^{1}(0, T), \rho_{-1}, \rho_{1} \in H^{1}(0, T)$ and

$$
\rho_{-1}(t)>0, \quad \rho_{1}(t)>0 \quad(t \in[0, T])
$$

(H4) $u_{0}(-1)=u_{-1}(0), u_{0}(1)=u_{1}(0), \rho_{-1}(0)=\rho_{0}(-1)$ and $\rho_{1}(0)=\rho_{0}(1)$.

Then, the initial and boundary value problem formed by (1.1), (1.2) and (1.5) admits a unique strong solution on $[0, T]$.

Moreover, the same conclusion (i.e., global existence and uniqueness of strong solutions) holds for the initial and boundary value problem formed by (1.1), (1.2) and (1.3), provided that we replace assumptions (H3) and (H4) respectively by

(H3') $u_{-1} \in H^{1}(0, T), \rho_{-1} \in H^{1}(0, T)$ and $\rho_{-1}(t)>0$ for every $t \in[0, T]$;

(H4') $u_{0}(-1)=u_{-1}(0), u_{0}(1)=0, \rho_{-1}(0)=\rho_{0}(-1)$.

Finally, the initial and boundary value problem formed by (1.1), (1.2) and (1.4) admits a unique strong solution on $[0, T]$ provided that the initial data satisfy $(H 1),(H 2)$ and $u_{0}(-1)=u_{0}(1)=0$.

The sense in which the term strong solution is understood in the above theorem will be made precise in the next section, but we can already mentioned that it includes the properties

$$
\begin{aligned}
u \in C\left([0, T] ; H^{1}(-1,1)\right), & \rho \in C\left(\bar{Q}_{-}\right) \cap C\left(\bar{Q}_{+}\right), \\
\inf _{-} \rho>0, & \inf _{\bar{Q}_{+}} \rho>0,
\end{aligned}
$$

where

$$
Q_{-}=\{(t, x) \in[0, T] \times[-1,1] \mid x<h(t)\}, \quad Q_{+}=\{(t, x) \in[0, T] \times[-1,1] \mid x<h(t)\} .
$$

As far as we know, the result in Theorem 1.1 is completely new because of the nonhomogeneous boundary conditions. In the case of homogeneous boundary conditions for the velocity, our statement in Theorem 1.1 differs from the corresponding result in [18] only by the fact that we require less regularity of the initial data.

To end this introduction we briefly describe the organization of the remaining part of this work. In Section 2 we describe a version of the change of variable to Lagrangian mass coordinates which is adapted to non homogeneous boundary conditions and we define the notion of strong solutions. The description of the governing equations using mass Lagrangian coordinates is then used in Section 3 to provide a detailed proof of the local in time existence of strong solutions. In Section 4 we go back to the Eulerian description of the governing equations and we establish some inequalities concerning the total mass and energy of the system. Section 5 provides, adapting the methodology introduced in Vaigant [20], pointwise and global estimates of the density field, which show, in particular, that cavitation cannot occur in finite time. The proof of Theorem 1.1 is given in Section 6. We end up with an Appendix where we justify, for the sake of completeness, some of the estimates on the linear transport equation with non homogeneous boundary conditions which have been used to prove local existence of strong solutions.

\section{Change of variables AND Definition of Strong SOlutions}

In this section we apply the Lagrangian mass change of coordinates to the system formed by (1.1), (1.2), (1.5). At the end of the section we describe describe how this change of variables adapts to tackle the problems (1.1), (1.2), (1.3) and (1.1), (1.2), (1.4). One of the advantages of this change of coordinates is that the positions of the piston becomes fixed but, as remarked in Belov [3], the extremities of the cylinder (which are fixed in the Eulerian framework) become mobile.

The passage to Lagrangian mass change of coordinates consists in replacing the space variable $x$ by $\xi=\Psi(t, x)$, where $\Psi(t, x)$ is the signed mass of the gas filling the domain between $h(t)$ and $x$ at instant $t$. More precisely, we set

$$
\Psi(t, x)=\int_{h(t)}^{x} \rho(t, \eta) \mathrm{d} \eta \quad(t \geqslant 0,-1 \leqslant x \leqslant 1) .
$$

Assume that $T>0, u_{-1}>0, u_{1}>0$ and that $(\rho, u)$ is a smooth enough solution of (1.1), (1.2) and (1.5) (this means, in particular, the function $\rho$ is positive and bounded away from zero) which is defined on the time 
interval $[0, T]$. Then, by a slight variation of the arguments in [3], it can be checked that for every $t \geqslant 0$ the map $x \mapsto \Psi(t, \cdot)$, with $\Psi$ defined in $(2.1)$, is a $C^{1}$ diffeomorphism which is strictly increasing from $(-1,1)$ to $\left(-\xi_{-1}(t), \xi_{1}(t)\right)$, where

$$
\xi_{-1}(t)=\int_{-1}^{h_{0}} \rho_{0}(\eta) \mathrm{d} \eta+\int_{0}^{t} \rho_{-1}(s) u_{-1}(s) \mathrm{d} s, \quad \xi_{1}(t)=\int_{h_{0}}^{1} \rho_{0}(\eta) \mathrm{d} \eta+\int_{0}^{t} \rho_{1}(s) u_{1}(s) \mathrm{d} s,
$$

for every $t \in[0, T]$. Moreover,

$$
\Psi(t, h(t))=0 \quad(t \in[0, T]) .
$$

For each $t \geqslant 0$ we denote by $\Phi(t, \cdot)=\Psi^{-1}(t, \cdot)$. The density in mass Lagrangian coordinates and the velocity field in Lagrangian mass coordinates are defined by

$$
\begin{array}{lll}
\widetilde{\rho}(t, \xi)=\rho(t, \Phi(t, \xi)), \quad \rho(t, x)=\widetilde{\rho}(t, \Psi(t, x)) & \left((t, x) \in Q_{h, T}, \quad(t, \xi) \in \widetilde{Q}_{\xi_{-1}, \xi_{1}, T}\right), \\
\widetilde{u}(t, \xi)=u(t, \Phi(t, \xi)), \quad u(t, x)=\widetilde{u}(t, \Psi(t, x)) & \left((t, x) \in Q_{h, T}, \quad(t, \xi) \in \widetilde{Q}_{\xi_{-1}, \xi_{1}, T}\right),
\end{array}
$$

where

$$
Q_{h, T}:=\{(t, x) \in(0, T) \times(-1,1) ; x \neq h(t)\},
$$

and

$$
\widetilde{Q}_{\xi_{-1}, \xi_{1}, T}:=\left\{(t, \xi) \in(0, T) \times \mathbb{R} ; \xi \in\left(-\xi_{-1}(t), \xi_{1}(t)\right), \quad \xi \neq 0 .\right\}
$$

Still according to [3], the system writes in the Lagrangian mass coordinates as

$$
\begin{array}{lr}
\partial_{t} \widetilde{\rho}+\widetilde{\rho}^{2} \partial_{\xi} \widetilde{u}=0 & \left((t, \xi) \in \widetilde{Q}_{\xi_{-1}, \xi_{1}, T}\right), \\
\partial_{t} \widetilde{u}-\partial_{\xi}\left(\widetilde{\rho}\left(\partial_{\xi} \widetilde{u}\right)\right)+\partial_{\xi}\left(\widetilde{\rho}^{\gamma}\right)=0 & \left((t, \xi) \in \widetilde{Q}_{\xi_{-1}, \xi_{1}, T}\right), \\
\widetilde{u}(t, 0)=\dot{h}(t) & (t \in[0, T]), \\
m \ddot{h}(t)=\left[\widetilde{\rho}\left(\partial_{\xi} \widetilde{u}\right)-\widetilde{\rho}^{\gamma}\right](t, 0) & (t \in[0, T]), \\
\widetilde{u}\left(t,-\xi_{-1}(t)\right)=u_{-1}(t), \widetilde{u}\left(t, \xi_{1}(t)\right)=-u_{1}(t) & (t \in[0, T]), \\
\widetilde{\rho}\left(t,-\xi_{-1}(t)\right)=\rho_{-1}(t) & (t \in[0, T]), \\
\widetilde{\rho}\left(t, \xi_{1}(t)\right)=\rho_{1}(t) & (t \in[0, T]), \\
\widetilde{\rho}(0, \xi):=\widetilde{\rho}_{0}(\xi)=\rho_{0}(\Phi(0, \xi)) & \left(\xi \in\left[-\xi_{-1}(0), \xi_{1}(0)\right] \backslash\{0\}\right), \\
\widetilde{u}(0, \xi):=\widetilde{u}_{0}(\xi)=u_{0}(\Phi(0, \xi)) & \left(\xi \in\left[-\xi_{-1}(0), \xi_{1}(0)\right] \backslash\{0\}\right),
\end{array}
$$

We have thus reached a situation in which the equations of the viscous gas hold in a domain which is still time variable, but with a prescribed variation (we do no longer have a free boundary problem).

We need a second change of variables in order to write the equations in a fixed domain. To this aim we define

$$
\Gamma(t, \xi)=\left\{\begin{array}{lll}
\frac{\xi}{\xi_{-1}(t)} & \text { for } & \xi \in\left[-\xi_{-1}(t), 0\right], \\
\frac{\xi}{\xi_{1}(t)} & \text { for } & \xi \in\left[0, \xi_{1}(t)\right] .
\end{array}\right.
$$

It is easy to verify that, for every $t \geqslant 0$, the mapping $\xi \mapsto \Gamma(\cdot, \xi)$ is a $C^{1}$ diffeomorphism from $\left(-\xi_{-1}(t), 0\right) \cup$ $\left(0, \xi_{1}(t)\right)$ onto $\Omega_{0}=(-1,0) \cup(0,1)$. We set $y=\Gamma(t, \xi)$ which yields

$$
\xi=\Gamma^{-1}(t, y)=\left\{\begin{array}{lll}
\xi_{-1}(t) y & \text { for } & y \in[-1,0] \\
\xi_{1}(t) y & \text { for } & y \in[0,1] .
\end{array}\right.
$$

Finally, we consider the specific volume $\zeta$ and the velocity field in the above system of coordinates, i.e.,

$$
\begin{array}{cc}
\zeta(t, y)=\left[\widetilde{\rho}\left(t, \Gamma^{-1}(t, y)\right)\right]^{-1}, \quad \widetilde{\rho}(t, \xi)=\left[\zeta(t, \Gamma((t, \xi))]^{-1} \quad\left((t, \xi) \in \widetilde{Q}_{\xi_{-1}, \xi_{1}, T},(t, y) \in Q_{0, T}\right),\right. \\
\bar{u}(t, y)=\widetilde{u}\left(t, \Gamma^{-1}(t, y)\right), \quad \widetilde{u}(t, \xi)=\bar{u}(t, \Gamma(t, \xi)) \quad\left((t, \xi) \in \widetilde{Q}_{\xi_{-1}, \xi_{1}, T},(t, y) \in Q_{0, T}\right) .
\end{array}
$$


Using the above change of coordinates, the system $(2.5 \mathrm{a})-(2.5 \mathrm{j})$ writes:

$$
\begin{array}{lr}
\partial_{t} \zeta+\beta \partial_{y} \zeta-\alpha \partial_{y} \bar{u}=0 & \text { in } Q_{0, T}, \\
\partial_{t} \bar{u}+\beta \partial_{y} \bar{u}-\alpha \partial_{y}\left(\frac{\alpha}{\zeta} \partial_{y} \bar{u}\right)+\alpha \partial_{y}\left(\frac{1}{\zeta^{\gamma}}\right)=0 & \text { in } Q_{0, T}, \\
\bar{u}(t, 0)=\dot{h}(t) & (t \in[0, T]), \\
m \ddot{h}=\left[\frac{\alpha}{\zeta} \partial_{y} \bar{u}-\frac{1}{\zeta^{\gamma}}\right](t, 0) & (t \in[0, T]), \\
\bar{u}(t,-1)=u_{-1}(t), \quad \bar{u}(t, 1)=-u_{1}(t) & (t \in[0, T]), \\
\zeta(t,-1)=\frac{1}{\rho_{-1}(t)} & (t \in(0, T)), \\
\zeta(t, 1)=\frac{1}{\rho_{1}(t)} & (t \in(0, T)), \\
\zeta(0, y)=\zeta_{0}(y), \quad \bar{u}(0, y)=\bar{u}_{0}(y) & (y \in[-1,1] \backslash\{0\}), \\
h(0)=h_{0}, \quad \dot{h}(0)=\ell_{0}, &
\end{array}
$$

where

$$
\alpha(t, y)=\left\{\begin{array}{ll}
\frac{1}{\xi_{-1}(t)} & \text { for } \quad y \in[-1,0), \\
\frac{1}{\xi_{1}(t)} & \text { for } \quad y \in(0,1]
\end{array}, \quad \beta(t, y)= \begin{cases}-\frac{y \dot{\xi}_{-1}(t)}{\xi_{-1}(t)} & \text { for } \quad y \in[-1,0) \\
-\frac{y \dot{\xi}_{1}(t)}{\xi_{1}(t)} & \text { for } \quad y \in(0,1]\end{cases}\right.
$$

and

$$
\zeta_{0}(y)=\left[\widetilde{\rho}_{0}\left(\Gamma^{-1}(0, y)\right)\right]^{-1}, \quad \bar{u}_{0}(y)=\widetilde{u}_{0}\left(\Gamma^{-1}(0, y)\right) .
$$

In what follows, we write for any $h \in(-1,1)$

$$
\Omega_{h}=(-1,1) \backslash\{h\} .
$$

We are now in a position to define the concept of strong solution of (1.1), (1.2) and (1.5).

Definition 2.1. Let $(h, \rho, u)$ be a triple of functions with $h:[0, \infty) \rightarrow(-1,1)$ and

$$
\rho(t, \cdot):(-1,1) \backslash\{h(t)\} \rightarrow(0, \infty), \quad u(t, \cdot):(-1,1) \backslash\{h(t)\} \rightarrow \mathbb{R} \quad(t \geqslant 0) .
$$

Let $T>0$. The triple $(h, \rho, u)$ is said to be a strong solution of (1.1), (1.2) and (1.5) on $[0, T]$ if

(1) $h \in H^{2}((0, T) ;(-1,1)), \quad u \in C\left([0, T] ; H^{1}(-1,1)\right), \rho \in C\left([0, T], L^{\infty}[-1,1]\right)$;

(2) $\rho(t, \cdot) \in H^{1}\left(\Omega_{h(t)}\right)$ for every $t \in[0, T]$ and there exists $M_{T}>0$ with

$$
\frac{1}{M_{T}} \leqslant \rho(t, x) \leqslant M_{T} \quad\left((t, x) \in Q_{h, T}\right) .
$$

(3) The function $\zeta$ and $\bar{u}$, defined in (2.6) and (2.7), are such that

$$
\begin{gathered}
\zeta \in C\left([0, T] ; H^{1}(-1,0) \cup H^{1}(0,1)\right) \cap H^{1}\left((0, T) ; L^{2}[-1,1]\right), \\
\bar{u} \in C\left([0, T] ; H^{1}[-1,1]\right) \cap H^{2,1}\left(Q_{0, T}\right),
\end{gathered}
$$

and the triplet $(h, \zeta, \bar{u})$ satisfies the system (2.8) in the strong sense.

In the above definition we have denoted, and we will continue to use this notation in the remaining part of this paper,

$$
H^{2,1}\left(Q_{0, T}\right):=L^{2}\left([0, T] ; H^{2}(-1,0) \cap H^{2}(0,1)\right) \cap H^{1}\left((0, T) ; L^{2}[-1,1]\right) .
$$


Remark 2.2. The change of variables and the definition in this section can be easily adapted to the cases in which instead of (1.5) we use the boundary conditions (1.3) or (1.4). More precisely, if we use the boundary conditions (1.3) then

$$
\xi_{1}(t)=\xi_{1}(0)=\int_{h_{0}}^{1} \rho_{0}(\eta) \mathrm{d} \eta, \quad \beta(t, y)=0 \quad \text { for } y \in(0,1], t \in[0, T]
$$

If we use the boundary conditions (1.4) then we retrieve the equations considered in [18], where

$\xi_{-1}(t)=\xi_{-1}(0)=\int_{-1}^{h_{0}} \rho_{0}(\eta) \mathrm{d} \eta, \quad \xi_{1}(t)=\xi_{1}(0)=\int_{h_{0}}^{1} \rho_{0}(\eta) \mathrm{d} \eta, \quad \beta(t, y)=0 \quad$ for $y \in[-1,1] \backslash\{0\}, t \in[0, T]$.

\section{Local in Time Existence And Uniqueness}

In this section $T \geqslant 1$ is a fixed (but arbitrary) number and we prove that (1.1), (1.2) and (1.5) admits a unique local in time strong solution, defined on some interval $\left[0, T_{*}\right]$, with $T_{*} \in(0, T]$. Moreover, we briefly explain why the employed methodology yields the same result for the problems (1.1), (1.2), (1.3) and (1.1), (1.2), (1.4). More precisely, denoting, for each $\varepsilon>0$,

$$
Y_{\varepsilon}=[-1+\varepsilon, 1-\varepsilon] \times\left(H^{1}\left(-1, h_{0}\right) \cap H^{1}\left(h_{0}, 1\right)\right) \times H^{1}(-1,1),
$$

the main result in this section can be stated as follows:

Theorem 3.1. Under the assumptions of Theorem 1.1, let $M>0$ be such that

$$
\begin{gathered}
\left\|u_{0}\right\|_{H^{1}(-1,1)}+\left\|\rho_{0}\right\|_{H^{1}\left(-1, h_{0}\right)}+\left\|\rho_{0}\right\|_{H^{1}\left(h_{0}, 1\right)}+\left|\ell_{0}\right| \leqslant M, \\
\frac{1}{M} \leqslant \rho_{0}(x) \leqslant M \quad\left(x \in[-1,1] \backslash\left\{h_{0}\right\}\right), \\
-1+\frac{1}{M} \leqslant h_{0} \leqslant 1-\frac{1}{M} .
\end{gathered}
$$

Moreover, we assume that $M$ satisfies

$$
\begin{gathered}
\left\|\rho_{-1}\right\|_{H^{1}(0, T)}+\left\|u_{-1}\right\|_{H^{1}(0, T)}+\left\|\rho_{1}\right\|_{H^{1}(0, T)}+\left\|u_{1}\right\|_{H^{1}(0, T)} \leqslant M, \\
u_{-1}(t), u_{1}(t), \rho_{-1}(t), \rho_{1}(t) \geqslant \frac{1}{M} \quad \text { for all } t \in[0, T] .
\end{gathered}
$$

Then there exists $T_{*} \in(0, T]$, depending only on $M$, such that (1.1), (1.2) and (1.5) admits a unique strong solution on $\left[0, T_{*}\right]$ and with the map

$$
\left[\begin{array}{l}
h_{0} \\
\rho_{0} \\
u_{0}
\end{array}\right] \mapsto\left[\begin{array}{l}
h \\
\rho \\
u
\end{array}\right]
$$

continuous from $Y_{1 / M}$ to $C\left(\left[0, T_{*}\right] ; Y_{\varepsilon}\right)$ for some $\varepsilon>0$, which also depends only on $M$.

The same conclusion holds for the systems (1.1), (1.2), (1.3) and (1.1), (1.2), (1.4), with obvious adaptations of the assumptions (3.2) and (3.3). 
The proof of the above theorem is based on a fixed point argument which is applied to the mass Lagrangian form (2.8) of the system. The fixed point procedure we adopt is based on the fact that (2.8) can be rewritten

$$
\begin{array}{lr}
\partial_{t} \zeta+\beta \partial_{y} \zeta-\alpha \partial_{y} \bar{u}=0 & \text { in } Q_{0, T}, \\
\partial_{t} \bar{u}-\alpha_{0} \partial_{y}\left(\frac{\alpha_{0}}{\zeta_{0}} \partial_{y} \bar{u}\right)=\mathcal{F}_{1}(\zeta, \bar{u}) & \text { in } Q_{0, T}, \\
\bar{u}(t, \pm 0)=\dot{h} & (t \in[0, T]), \\
m \ddot{h}=\left[\frac{\alpha_{0}}{\zeta_{0}} \partial_{y} \bar{u}\right](t, 0)+\mathcal{F}_{2}(\zeta, \bar{u}) & (t \in[0, T]), \\
\bar{u}(t,-1)=u_{-1}(t), \quad \bar{u}(t, 1)=-u_{1}(t) & (t \in[0, T]), \\
\zeta(t,-1)=\frac{1}{\rho_{-1}(t)} & (t \in[0, T]), \\
\zeta(t, 1)=\frac{1}{\rho_{1}(t)} & (t \in[0, T]), \\
\zeta(0, y):=\zeta_{0}(y), \quad \bar{u}(0, y)=\bar{u}_{0}(y) & (y \in[-1,1] \backslash\{0\}), \\
h(0)=h_{0}, \quad \dot{h}(0)=\ell_{0}, &
\end{array}
$$

where $\alpha_{0}=\alpha(0, \cdot), Q_{0, T}$ has been defined in (2.4) and

$$
\begin{aligned}
& \mathcal{F}_{1}(\zeta, \bar{u})=\alpha \partial_{y}\left(\frac{\alpha}{\zeta} \partial_{y} \bar{u}\right)-\alpha_{0} \partial_{y}\left(\frac{\alpha_{0}}{\zeta_{0}} \partial_{y} \bar{u}\right)-\alpha \partial_{y}\left(\frac{1}{\zeta^{\gamma}}\right)-\beta \partial_{y} \bar{u} \\
& \mathcal{F}_{2}(\zeta, \bar{u})=\left[\left(\frac{\alpha}{\zeta}-\frac{\alpha_{0}}{\zeta_{0}}\right)\left(\partial_{y} \bar{u}\right)\right](t, 0)-\left[\frac{1}{\zeta^{\gamma}}\right](t, 0) .
\end{aligned}
$$

The idea is to replace in the above system $\mathcal{F}_{1}$ and $\mathcal{F}_{2}$ by given source terms $f_{1}$ and $f_{2}$. We then obtain a linear system that can be studied by splitting it into a parabolic equation for the velocities (fluid and particle velocities) and a transport equation for the specific volume. The proof of our local in time existence and uniqueness result is then concluded by applying Banach's fixed point theorem.

In this section, $K$ denotes a positive constant that may depend on $M$ and that may change from line to line.

Before beginning to apply the methodology described above we note that we have the following result, which can be checked in an obvious way, so we state it without proof.

Lemma 3.2. Let $\left(\rho_{0}, u_{0}, h_{0}, \ell_{0}, \rho_{-1}, \rho_{1}, u_{-1}, u_{1}\right)$ and $M$ be as in Theorem 3.1. Then the function $\zeta_{0}$ and $\bar{u}_{0}$ which have been defined in (2.10) satisfy

$$
\begin{gathered}
\zeta_{0} \in H^{1}(-1,0) \cap H^{1}(0,1), \quad \bar{u}_{0} \in H^{1}(-1,1), \\
\bar{u}_{0}(-1)=u_{-1}(0), \quad \bar{u}_{0}(0)=\ell_{0}, \quad \bar{u}_{0}(1)=u_{1}(0), \\
\left\|\bar{u}_{0}\right\|_{H^{1}(-1,1)}+\left\|\zeta_{0}\right\|_{H^{1}\left(\Omega_{0}\right)} \leqslant K, \\
\frac{1}{K} \leqslant \zeta_{0}(y) \leqslant K \quad(y \in[-1,1] \backslash\{0\}),
\end{gathered}
$$

where $K>0$ depends only on $M$. 
The first step in proving Theorem 3.1 is the following result on the linear parabolic type system

$$
\begin{array}{lr}
\partial_{t} \bar{u}-\alpha_{0} \partial_{y}\left(\frac{\alpha_{0}}{\zeta_{0}} \partial_{y} \bar{u}\right)=f_{1} & \text { in } Q_{0, T}, \\
\bar{u}(t, 0)=\dot{h}(t) & (t \in[0, T]), \\
m \ddot{h}(t)=\left[\frac{\alpha_{0}}{\zeta_{0}} \partial_{y} \bar{u}\right](t, 0)+f_{2}(t) & (t \in[0, T]), \\
\bar{u}(t,-1)=u_{-1}(t), \quad \bar{u}(t, 1)=-u_{1}(t) & (t \in[0, T]), \\
\bar{u}(0, y)=\bar{u}_{0}(y) & (y \in[-1,1] \backslash\{0\}), \\
h(0)=h_{0}, \quad \dot{h}(0)=\ell_{0}, &
\end{array}
$$

where $f_{1}$ and $f_{2}$ are given source terms. Note that, using a term often employed in the study of fluid-structure interactions, we can say the considered system is "monolithic". This means that this linearization preserves the coupling of the equations of the fluid and of the structure. This contrasts with the approach used in previous works (see, for instance, [18]) where the corresponding step consists in first solving uncoupled parabolic equations on each side of the piston, with non homogeneous boundary conditions at $y=0$. The choice of this monolithic proof scheme is essential in our approach for obtaining the local existence result in Theorem 3.1 for initial data less regular than in the previous literature.

For the remaining part of this section we denote, for each $T_{*} \in(0, T]$,

$$
\mathcal{B}_{T_{*}}=\left\{\left[\begin{array}{l}
f_{1} \\
f_{2}
\end{array}\right] \in L^{2}\left(Q_{0, T_{*}}\right) \times L^{2}\left[0, T_{*}\right] \mid\left\|f_{1}\right\|_{L^{2}\left(Q_{0, T_{*}}\right)}+\left\|f_{2}\right\|_{L^{2}\left[0, T_{*}\right]} \leqslant 1\right\},
$$

where we recall from (2.4) that

$$
Q_{0, T_{*}}=\left\{(t, x) \in\left(0, T_{*}\right) \times(-1,1) ; x \neq h(t)\right\} .
$$

Proposition 3.3. Let $T \geqslant 1$ be the number fixed at the beginning of this section. Let $\left(\rho_{0}, u_{0}, h_{0}, \ell_{0}, u_{-1}, u_{1}\right)$ and $M$ be as in Theorem 3.1 and let $\left(\zeta_{0}, \bar{u}_{0}\right)$ be defined as in (2.10). Then for every $\left[\begin{array}{l}f_{1} \\ f_{2}\end{array}\right] \in \mathcal{B}_{T}$ the system (3.8) admits a unique strong solution on $[0, T]$. Moreover, there exists $K_{1}>0$, depending only on $M$, such that

$$
\|\bar{u}\|_{H^{2,1}\left(Q_{0, T}\right)}+\|\bar{u}\|_{C\left([0, T] ; H^{1}(-1,1)\right)}+\|h\|_{H^{2}(0, T)} \leqslant K_{1},
$$

where the space $H^{2,1}\left(Q_{0, T}\right)$ has been defined in (2.12).

The above result can be proved modulo an obvious adaptation of the proof of the corresponding result for $\alpha_{0}=\zeta_{0}=1, u_{-1}=u_{1}=0$ which has been given in Proposition 3.3 from [6], so that we omit the details.

Corollary 3.4. With the notation and under the assumptions of Proposition 3.3 there exists $K>0$, depending only on $M$, such that

$$
\left\|\partial_{y} \bar{u}\right\|_{L^{2}\left(\left[0, T_{*}\right] ; L^{\infty}\left(\Omega_{0}\right)\right)} \leqslant K T_{*}^{1 / 8} \quad\left(T_{*} \in(0, T]\right),
$$

where (we recall from (2.11))

$$
\Omega_{0}=(-1,1) \backslash\{0\}
$$

Proof. We first note that

$$
\left\|\partial_{y} \bar{u}(t, \cdot)\right\|_{L^{\infty}\left(\Omega_{0}\right)} \leqslant K\left\|\partial_{y} \bar{u}(t, \cdot)\right\|_{H^{1}\left(\Omega_{0}\right)}^{3 / 4}\left\|\partial_{y} \bar{u}(t, \cdot)\right\|_{L^{2}\left(\Omega_{0}\right)}^{1 / 4} \quad(t \in(0, T)),
$$

which yields

$$
\int_{0}^{T_{*}}\left\|\partial_{y} \bar{u}(t, \cdot)\right\|_{L^{\infty}\left(\Omega_{0}\right)}^{2} \mathrm{~d} t \leqslant K\left\|\partial_{y} \bar{u}\right\|_{L^{\infty}\left([0, T] ; L^{2}\left(\Omega_{0}\right)\right)}^{1 / 2} \int_{0}^{T_{*}}\left\|\partial_{y} \bar{u}(t, \cdot)\right\|_{H^{1}\left(\Omega_{0}\right)}^{3 / 2} \mathrm{~d} t .
$$

The two above estimates and Hölder's inequality imply that

$$
\left\|\partial_{y} \bar{u}\right\|_{L^{2}\left(\left[0, T_{*}\right] ; L^{\infty}\left(\Omega_{0}\right)\right)} \leqslant K T_{*}^{1 / 8}\left\|\partial_{y} \bar{u}\right\|_{L^{2}\left([0, T] ; H^{1}\left(\Omega_{0}\right)\right)}^{3 / 4}\left\|\partial_{y} \bar{u}\right\|_{L^{\infty}\left([0, T] ; L^{2}\left(\Omega_{0}\right)\right)}^{1 / 4} \quad\left(T_{*} \in(0, T]\right) .
$$

The conclusion (3.12) follows now from the above inequality and (3.11). 
The second step in proving Theorem 3.1 is to use the velocity field $\bar{u}$ constructed in Proposition 3.3 to solve an initial and boundary value problem for the transport equation. The choice of the boundary conditions for this problem depends on the choice (among (1.3), (1.4) or (1.5)) of the boundary conditions in Theorem 3.1. As already asserted at the beginning of this section, we provide detailed proof for the boundary condition (1.5), whence in the case when $\beta(t,-1)$ and $\beta(t, 1)$ are positive and bounded away from zero (recall that $\beta$ has been defined in $(2.9))$. In this case we consider the system:

$$
\left\{\begin{array}{lr}
\partial_{t} \zeta(t, y)+\beta(t, y) \partial_{y} \zeta(t, y)=\alpha(t, y)\left(\partial_{y} \bar{u}\right)(t, y) & (t, y) \in Q_{0, T}, \\
\zeta(t,-1)=\frac{1}{\rho_{-1}(t)} & (t \in[0, T]), \\
\zeta(t, 1)=\frac{1}{\rho_{1}(t)} & (t \in[0, T]), \\
\zeta(0, y)=\zeta_{0}(y) & (y \in[-1,1] \backslash\{0\}),
\end{array}\right.
$$

with $\alpha$ and $\beta$ defined in (2.9). Our methods easily adapt to the boundary conditions (1.3) or (1.4), the main change being that, instead of using Proposition A.2 (as in the proof of Proposition 3.5 below) we use (at least once) Proposition A.1.

Proposition 3.5. Under the assumptions of Proposition 3.3 let $\bar{u}$ be the solution on $[0, T]$, with $T \geqslant 1$ fixed at the beginning of the section, of (3.8) constructed in the above mentioned proposition. Then the system (3.16) admits a unique solution $\zeta \in C\left([0, T] ; H^{1}\left(\Omega_{0}\right)\right) \cap C^{1}\left([0, T] ; L^{2}[-1,1]\right)$.

Moreover, there exists $K>0$, depending only on $M$, such that

$$
\|\zeta\|_{C\left(\left[0, T_{*}\right] ; H^{1}\left(\Omega_{0}\right)\right)}+\left\|\partial_{t} \zeta\right\|_{C\left(\left[0, T_{*}\right] ; L^{2}[-1,1]\right)} \leqslant K \quad\left(T_{*} \in(0,1]\right) .
$$

Finally, there exist $\widetilde{T} \in(0,1]$ and $K>0$, both depending only on $M$, such that

$$
\begin{array}{cl}
\frac{1}{K} \leqslant \zeta(t, y) \leqslant K & (t \in[0, \widetilde{T}]), \\
\left\|\frac{1}{\zeta}-\frac{1}{\zeta_{0}}\right\|_{L^{\infty}\left(Q_{\left.0, T_{*}\right)}\right.} \leqslant K T_{*}^{1 / 4} & \left(T_{*} \in[0, \widetilde{T}]\right) .
\end{array}
$$

Proof. We first note that since, by the definition (2.9), we have $\beta(t, 0)=0$, the system (3.16) splits in two uncoupled initial and boundary value problems: the first one on $[-1,0]$ and the second one on $[0,1]$. We prove below the required estimates only for $y \in[-1,0]$, the proof of the corresponding estimates for $y \in[0,1]$ being completely similar. This is done below by a direct application of Proposition A.2 from Section A, with $w=\beta$. To check that $\beta$ satisfies the assumptions in Proposition A.2, we first notice that

$$
\beta(t,-1)=\frac{\rho_{-1}(t) u_{-1}(t)}{\xi_{-1}(t)}>0 \text { and } \beta(t, 0)=0 \quad(t \in[0, T]) .
$$

We next note that from (2.2) and (3.1)-(3.3) it follows that

$$
\begin{array}{cc}
\frac{1}{M}\left(h_{0}+1\right) \leqslant \xi_{-1}(t) \leqslant M\left(h_{0}+1\right)+M^{2} & \left(t \in\left[0, T_{*}\right], T_{*} \in(0,1]\right), \\
\frac{1}{M}\left(1-h_{0}\right) \leqslant \xi_{1}(t) \leqslant M\left(1-h_{0}\right)+M^{2} & \left(t \in\left[0, T_{*}\right], T_{*} \in(0,1]\right) .
\end{array}
$$

Combining the two above inequalities, (3.20) and (3.1) we obtain

$$
\begin{gathered}
\|\beta(\cdot,-1)\|_{L^{\infty}\left[0, T_{*}\right]} \leqslant K, \\
\|\beta\|_{C\left(\left[0, T_{*}\right] ; H^{1}(-1,0)\right) \cap L^{2}\left(\left[0, T_{*}\right] ; H^{2}(-1,0)\right)} \leqslant K,
\end{gathered}
$$

where $K$ is a constant depending only on $M$. On the other hand, using (3.2) and (3.3) we have

$$
\left\|\frac{1}{\rho_{-1}}\right\|_{H^{1}\left(0, T_{*}\right)} \leqslant K
$$


where $K$ is a constant depending only on $M$. The estimates (3.23)-(3.25), together with Corollary 3.4 allow us to apply Proposition A.2 and thus to obtain

$$
\|\zeta\|_{C\left(\left[0, T_{*}\right] ; H^{1}(-1,0)\right)}+\left\|\partial_{t} \zeta\right\|_{C\left(\left[0, T_{*}\right] ; L^{2}[-1,0]\right)} \leqslant K,
$$

where $K$ depends only on $M$. In this way we obtain the existence and uniqueness of a solution $\zeta$ satisfying (3.17).

In order to prove (3.18) and (3.19) it suffices to note that from (3.17) and a simple interpolation argument, there exists a constant $K$ such that, for every $t \in\left[0, T_{*}\right]$ we have

$$
\begin{aligned}
& \left\|\zeta(t, \cdot)-\zeta_{0}\right\|_{L^{\infty}\left(\Omega_{0}\right)} \leqslant K\left\|\zeta-\zeta_{0}\right\|_{L^{\infty}\left(\left[0, T_{*}\right] ; H^{1}\left(\Omega_{0}\right)\right)}^{3 / 4}\left\|\zeta-\zeta_{0}\right\|_{L^{\infty}\left(\left[0, T_{*}\right] ; L^{2}\left(\Omega_{0}\right)\right)}^{1 / 4} \\
& \leqslant K T_{*}^{1 / 4}\left\|\zeta-\zeta_{0}\right\|_{C\left(\left[0, T_{*}\right] ; H^{1}\left(\Omega_{0}\right)\right)}^{3 / 4}\|\zeta\|_{C^{1}\left(\left[0, T_{*}\right] ; L^{2}\left(\Omega_{0}\right)\right)}^{1 / 4} \\
& \leqslant K T_{*}^{1 / 4}
\end{aligned}
$$

Lemma 3.6. For $T_{*} \in(0, \widetilde{T}]$, where $\widetilde{T}$ is the constant in Proposition 3.5, let $\mathcal{B}_{T_{*}}$ be the ball defined by (3.9). Let $\left(\rho_{0}, u_{0}, h_{0}, \ell_{0}, u_{-1}, u_{1}\right)$ and $M$ be as in Theorem 3.1. Let $\left(\zeta_{0}, \bar{u}_{0}\right)$ be defined in $(2.10)$. Given $\left[\begin{array}{l}f_{1} \\ f_{2}\end{array}\right] \in \mathcal{B}_{T_{*}}$, let $(h, \bar{u})$ (respectively $\zeta$ ) be the solution of (3.8) (respectively of (3.16)) on $\left[0, T_{*}\right]$ constructed in Proposition 3.3 (respectively in Proposition 3.5).

Then there exists a constant $K_{2}>0$, depending only on $M$, such that

$$
\begin{array}{ll}
\left\|\mathcal{F}_{1}(\bar{u}, \zeta)\right\|_{L^{2}\left(Q_{0, T_{*}}\right)} \leqslant K_{2} T_{*}^{1 / 8} & \left(\left[\begin{array}{l}
f_{1} \\
f_{2}
\end{array}\right] \in \mathcal{B}_{T_{*}}\right), \\
\left\|\mathcal{F}_{2}(\bar{u}, \zeta)\right\|_{L^{2}\left[0, T_{*}\right]} \leqslant K_{2} T_{*}^{1 / 4} & \left(\left[\begin{array}{l}
f_{1} \\
f_{2}
\end{array}\right] \in \mathcal{B}_{T_{*}}\right),
\end{array}
$$

where $\mathcal{F}_{1}, \mathcal{F}_{2}$ have been defined in (3.6) and (3.7).

Proof. We first note that, using the definition (2.9) of $\alpha$, together with (3.21) and (3.22), one can check that there exists a constant $K$, depending only on $M$, such that

$$
\left\|\alpha-\alpha_{0}\right\|_{L^{\infty}\left(Q_{0, T_{*}}\right)} \leqslant K_{2} T_{*} .
$$

To estimate the sum of the first two terms of $\mathcal{F}_{1}$ we note that

$$
\alpha^{2} \partial_{y}\left(\frac{1}{\zeta} \partial_{y} \bar{u}\right)-\alpha_{0}^{2} \partial_{y}\left(\frac{1}{\zeta_{0}} \partial_{y} \bar{u}\right)=\left(\alpha^{2}-\alpha_{0}^{2}\right) \partial_{y}\left(\frac{1}{\zeta} \partial_{y} \bar{u}\right)+\alpha_{0}^{2} \partial_{y}\left(\left(\frac{1}{\zeta}-\frac{1}{\zeta_{0}}\right) \partial_{y} \bar{u}\right) .
$$

Using (3.11), (3.21), (3.22), (3.17), (3.18) and (3.29) it follows that there exists $K_{2}>0$ depending only on $M$, such that the first term in the right hand side of the above formula satisfies, for every $T_{*} \in(0, \widetilde{T})$,

$$
\left\|\left(\alpha^{2}-\alpha_{0}^{2}\right) \partial_{y}\left(\frac{1}{\zeta} \partial_{y} \bar{u}\right)\right\|_{L^{2}\left(Q_{\left.0, T_{*}\right)}\right.} \leqslant K_{2}\left\|\alpha-\alpha_{0}\right\|_{L^{\infty}\left(Q_{0, T_{*}}\right)}\left(\left\|\frac{1}{\zeta} \partial_{y y} \bar{u}\right\|_{L^{2}\left(Q_{0, T_{*}}\right)}+\left\|\frac{\partial_{y} \zeta}{\zeta^{2}} \partial_{y} \bar{u}\right\|_{L^{2}\left(Q_{\left.0, T_{*}\right)}\right)}\right) \leqslant K_{2} T_{*} .
$$

Similarly, using again (3.11), (3.17), (3.26), (3.18) and (3.12), it is not difficult to check that there exists $K_{2}>0$, depending only on $M$, such that the second term in the right hand side of (3.30) satisfies

$$
\begin{aligned}
\left\|\alpha_{0}^{2} \partial_{y}\left(\left(\frac{1}{\zeta}-\frac{1}{\zeta_{0}}\right) \partial_{y} \bar{u}\right)\right\|_{L^{2}\left(Q_{0, T_{*}}\right)} \leqslant K_{2}\left(\left\|\frac{1}{\zeta}-\frac{1}{\zeta_{0}}\right\|_{L^{\infty}\left(Q_{0, T_{*}}\right)}\left\|\partial_{y y} \bar{u}\right\|_{L^{2}\left(Q_{0, T_{*}}\right)}\right. \\
\left.\quad+\left(\left\|\frac{\partial_{y} \zeta}{\zeta^{2}}\right\|_{L^{\infty}\left(\left[0, T_{*}\right] ; L^{2}\left(\Omega_{0}\right)\right.}+\left\|\frac{\partial_{y} \zeta_{0}}{\zeta_{0}^{2}}\right\|_{L^{\infty}\left(\left[0, T_{*}\right] ; L^{2}\left(\Omega_{0}\right)\right.}\right)\left\|\partial_{y} \bar{u}\right\|_{L^{2}\left(\left[0, T_{*}\right] ; L^{\infty}\left(\Omega_{0}\right)\right.}\right) \leqslant K_{2} T_{*}^{1 / 8}
\end{aligned}
$$


Using (3.18), (3.17) and (3.11), the last two terms in the definition (3.6) of $\mathcal{F}_{1}$ satisfy

$$
\begin{aligned}
& \left\|\alpha \partial_{y}\left(\frac{1}{\zeta^{\gamma}}\right)+\beta \partial_{y} \bar{u}\right\|_{L^{2}\left(Q_{0, T_{*}}\right)} \leqslant K_{2}\left(\left\|\partial_{y} \zeta\right\|_{L^{2}\left(Q_{0, T_{*}}\right)}+\left\|\partial_{y} \bar{u}\right\|_{L^{2}\left(Q_{0}, T_{*}\right)}\right) \\
& \leqslant K_{2} \sqrt{T_{*}}\left(\left\|\partial_{y} \zeta\right\|_{L^{\infty}\left(\left[0, T_{*}\right] ; L^{2}\left(\Omega_{0}\right)\right)}+\left\|\partial_{y} \bar{u}\right\|_{\left.L^{\infty}\left(\left[0, T_{*}\right] ; L^{2}\left(\Omega_{0}\right)\right)\right)}\right) \leqslant K_{2} \sqrt{T_{*}},
\end{aligned}
$$

where $K_{3}>0$ depends only on $M$.

By combining (3.31)-(3.33) we obtain the first claimed estimate (3.27).

In order to prove $(3.27)$, we first use $(3.26),(3.18)$ and $(3.29)$ to obtain that the first term in the definition (3.7) of $\mathcal{F}_{2}$ satisfies

$$
\begin{aligned}
& \left\|\left[\left(\frac{\alpha}{\zeta}-\frac{\alpha_{0}}{\zeta_{0}}\right)\left(\partial_{y} \bar{u}\right)\right](t, 0)\right\|_{L^{2}\left[0, T_{*}\right]} \\
& \leqslant K_{2}\left\|\frac{\alpha}{\zeta}-\frac{\alpha_{0}}{\zeta_{0}}\right\|_{L^{\infty}\left(Q_{\left.0, T_{*}\right)}\right.}\|\bar{u}\|_{H^{2,1}\left(Q_{0, T_{*}}\right)} \\
& \leqslant K_{2}\|\bar{u}\|_{H^{2,1}\left(Q_{\left.0, T_{*}\right)}\right.}\left(\left\|\frac{\alpha-\alpha_{0}}{\zeta}\right\|_{L^{\infty}\left(Q_{\left.0, T_{*}\right)}\right.}+\left\|\alpha_{0}\left(\frac{1}{\zeta}-\frac{1}{\zeta_{0}}\right)\right\|_{L^{\infty}\left(Q_{0, T_{*}}\right)}\right) \\
& \leqslant K_{2} T_{*}^{1 / 4},
\end{aligned}
$$

where $K_{2}>0$ depends only on $M$. Using next (3.17), it is easy to verify that there exists $K_{2}>0$, depending only on $M$, such that the last term of the definition (3.7) of $\mathcal{F}_{2}$ satisfies

$$
\left\|\left[\frac{1}{\zeta^{\gamma}}\right](\cdot, 0)\right\|_{L^{2}\left[0, T_{*}\right]} \leqslant \sqrt{T_{*}}\left\|\left[\frac{1}{\zeta^{\gamma}}\right](\cdot, 0)\right\|_{L^{\infty}\left[0, T_{*}\right]} \leqslant K_{2} \sqrt{T_{*}} .
$$

The two inequalities above clearly imply the second claimed estimate (3.28).

Lemma 3.7. For $T_{*} \in(0, \widetilde{T}]$, where $\widetilde{T}$ is the constant in Proposition 3.5 , let $\mathcal{B}_{T_{*}}$ be the ball defined by (3.9). Let $\left(\rho_{0}, u_{0}, h_{0}, \ell_{0}, u_{-1}, u_{1}\right)$ and $M$ be as in Theorem 3.1. Let $\left(\zeta_{0}, \bar{u}_{0}\right)$ be defined in (2.10). Given $\left[\begin{array}{l}f_{1}^{j} \\ f_{2}^{j}\end{array}\right] \in \mathcal{B}_{T_{*}}$, for $j=1,2$, let $\left(h^{j}, \bar{u}^{j}\right)$ (respectively by $\zeta^{j}$ ) the solution of (3.8) (respectively of (3.16)) corresponding to the source term $\left(f_{1}^{j}, f_{2}^{j}\right)$ (respectively to the coefficient $\left.\bar{u}^{j}\right)$. Then there exists a constant $K_{3}>0$, depending only on $M$, such that

$$
\begin{array}{cc}
\left\|\mathcal{F}_{1}\left(\zeta^{1}, \bar{u}^{1}\right)-\mathcal{F}_{1}\left(\zeta^{2}, \bar{u}^{2}\right)\right\|_{L^{2}\left(Q_{0, T_{*}}\right)}+\left\|\mathcal{F}_{2}\left(\zeta^{1}, \bar{u}^{1}\right)-\mathcal{F}_{2}\left(\zeta^{2}, \bar{u}^{2}\right)\right\|_{L^{2}\left[0, T_{*}\right]} & \\
\leqslant K_{3} T_{*}^{1 / 8}\left(\left\|f_{1}^{1}-f_{1}^{2}\right\|_{L^{2}\left(Q_{0, T_{*}}\right)}+\left\|f_{2}^{1}-f_{2}^{2}\right\|_{L^{2}\left[0, T_{*}\right]}\right) & \left(\left[\begin{array}{l}
f_{1}^{1} \\
f_{2}^{1}
\end{array}\right],\left[\begin{array}{l}
f_{1}^{2} \\
f_{2}^{2}
\end{array}\right] \in \mathcal{B}_{T_{*}}\right),
\end{array}
$$

where $\mathcal{F}_{1}, \mathcal{F}_{2}$ have been defined in (3.6) and (3.7).

Proof. Setting

$$
\breve{\zeta}=\zeta^{1}-\zeta^{2}, \quad \breve{u}=u^{1}-u^{2}, \quad \breve{h}=h^{1}-h^{2}
$$


we have

$$
\begin{array}{lr}
\partial_{t} \breve{u}-\alpha_{0} \partial_{y}\left(\frac{\alpha_{0}}{\zeta_{0}} \partial_{y} \breve{u}\right)=f_{1}^{1}-f_{1}^{2} & \text { in } Q_{0, T_{*},}, \\
\breve{u}(t, 0)=\dot{\breve{h}}(t) & \left(t \in\left[0, T_{*}\right]\right), \\
m \ddot{h}(t)=\left[\frac{\alpha_{0}}{\zeta_{0}} \partial_{y} \breve{u}\right](t, 0)+f_{2}^{1}(t)-f_{2}^{2}(t) & \left(t \in\left[0, T_{*}\right]\right), \\
\breve{u}(t,-1)=0, \quad \breve{u}(t, 1)=0 & t \in\left[0, T_{*}\right], \\
\breve{u}(0, y)=0 & y \in[-1,1] \backslash\{0\}, \\
\breve{h}(0)=0, \quad \dot{\breve{h}}(0)=0, &
\end{array}
$$

together with

$$
\begin{array}{lr}
\partial_{t} \breve{\zeta}+\beta \partial_{y} \breve{\zeta}=\alpha\left(\partial_{y} \breve{u}\right) & \text { in } Q_{0, T_{*},} \\
\breve{\zeta}(\cdot,-1)=0 & \left(t \in\left[0, T_{*}\right]\right), \\
\breve{\zeta}(\cdot, 1)=0 & \left(t \in\left[0, T_{*}\right]\right), \\
\breve{\zeta}(0, y)=0 & (y \in[-1,1] \backslash\{0\}) .
\end{array}
$$

Using (3.11) we obtain

$$
\begin{aligned}
\|\breve{u}\|_{L^{2}\left(\left[0, T_{*}\right] ; H^{2}\left(\Omega_{0}\right)\right)}+\|\breve{u}\|_{H^{1}\left(\left(0, T_{*}\right) ; L^{2}[-1,1]\right)}+\|\breve{u}\|_{C\left(\left[0, T_{*}\right] ; H^{1}(-1,1)\right)}+\|\breve{h}\|_{H^{2}\left(0, T_{*}\right)} \\
\quad \leqslant K_{3}\left(\left\|f_{1}^{1}-f_{1}^{2}\right\|_{L^{2}\left(Q_{0, T_{*}}\right)}+\left\|f_{2}^{1}-f_{2}^{2}\right\|_{L^{2}\left[0, T_{*}\right]}\right)
\end{aligned}
$$

where $K_{3}$ that depends only on $M$.

On the other hand, by applying Proposition A.2 from Section A (as in the proof of Proposition 3.5) we deduce that

$$
\|\breve{\zeta}\|_{C\left(\left[0, T_{*}\right] ; H^{1}\left(\Omega_{0}\right)\right)}+\left\|\partial_{t} \breve{\zeta}\right\|_{C\left(\left[0, T_{*}\right] ; L^{2}[-1,1]\right)} \leqslant K_{3}\left(\|\breve{u}\|_{L^{2}\left(\left[0, T_{*}\right] ; H^{2}\left(\Omega_{0}\right)\right)}+\|\breve{u}\|_{C\left(\left[0, T_{*}\right] ; H^{1}(-1,1)\right)}\right) .
$$

where the positive constant $K_{3}$ depends only on $M$.

Moreover, we have

$$
\begin{array}{r}
\mathcal{F}_{1}\left(\zeta^{1}, \bar{u}^{1}\right)-\mathcal{F}_{1}\left(\zeta^{2}, \bar{u}^{2}\right)=\left(\alpha-\alpha_{0}\right) \partial_{y}\left(\frac{\alpha}{\zeta^{1}} \partial_{y} \breve{u}\right)+\alpha \partial_{y}\left(\alpha\left(\frac{1}{\zeta^{1}}-\frac{1}{\zeta^{2}}\right) \partial_{y} \breve{u}\right)+\alpha_{0} \partial_{y}\left(\alpha\left(\frac{1}{\zeta^{1}}-\frac{1}{\zeta_{0}}\right) \partial_{y} \breve{u}\right) \\
-\alpha \partial_{y}\left(\frac{1}{\left(\zeta^{1}\right)^{\gamma}}-\frac{1}{\left(\zeta^{2}\right)^{\gamma}}\right)-\beta \partial_{y} \breve{u}
\end{array}
$$

and

$$
\mathcal{F}_{2}\left(\zeta^{1}, \bar{u}^{1}\right)-\mathcal{F}_{2}\left(\zeta^{2}, \bar{u}^{2}\right)=\left[\left(\frac{\alpha}{\zeta^{1}}-\frac{\alpha_{0}}{\zeta_{0}}\right)\left(\partial_{y} \breve{u}\right)\right](t, 0)+\left[\left(\frac{\alpha}{\zeta^{1}}-\frac{\alpha}{\zeta^{2}}\right)\left(\partial_{y} \bar{u}_{2}\right)\right](t, 0)-\left[\frac{1}{\zeta^{\gamma}}\right](t, 0) .
$$

Using (3.21), (3.22),(3.17), (3.18) and (3.29) it follows that there exists $K_{3}=K_{3}(M)>0$ such that

$$
\left\|\left(\alpha-\alpha_{0}\right) \partial_{y}\left(\frac{\alpha}{\zeta^{1}} \partial_{y} \breve{u}\right)\right\|_{L^{2}\left(Q_{0, T_{*}}\right)} \leqslant K_{3} T_{*}\left(\left\|\partial_{y y} \breve{u}\right\|_{L^{2}\left(Q_{0, T_{*}}\right)}+\left\|\left(\partial_{y} \zeta_{1}\right)\left(\partial_{y} \breve{u}\right)\right\|_{L^{2}\left(Q_{0, T_{*}}\right)}\right) \leqslant K_{3} T_{*}\|\breve{u}\|_{H^{2,1}\left(Q_{0, T_{*}}\right)} .
$$


Similarly, using again (3.17), (3.26), (3.18) and (3.12), the second term of (3.39) satisfies

$$
\begin{aligned}
& \left.\left\|\alpha \partial_{y}\left(\alpha\left(\frac{1}{\zeta^{1}}-\frac{1}{\zeta^{2}}\right) \partial_{y} \breve{u}\right)\right\|_{L^{2}\left(Q_{\left.0, T_{*}\right)}\right.} \leqslant K_{3}\left(\left\|\frac{1}{\zeta^{1}}-\frac{1}{\zeta_{2}}\right\|_{L^{\infty}\left(Q_{0, T_{*}}\right)}\left\|\partial_{y y} \breve{u}\right\|_{L^{2}\left(Q_{0, T_{*}}\right)}\right)\left\|\partial_{y} \breve{u}\right\|_{L^{2}\left(\left[0, T_{*}\right] ; L^{\infty}\left(\Omega_{0}\right)\right)}\right) \leq K_{3} T_{*}^{1 / 8}\|\breve{u}\|_{H^{2,1}\left(Q_{0, T_{*}}\right)} \\
& +\left(\left\|\frac{\partial_{y} \zeta^{1}}{\left(\zeta^{1}\right)^{2}}\right\|_{L^{\infty}\left(\left[0, T_{*}\right] ; L^{2}\left(\Omega_{0}\right)\right)}+\left\|\frac{\partial_{y} \zeta^{2}}{\left(\zeta^{2}\right)^{2}}\right\|_{L^{\infty}\left(\left[0, T_{*}\right] ; L^{2}\left(\Omega_{0}\right)\right)}\right)
\end{aligned}
$$

where $K_{3}=K_{3}(M)>0$. The other terms in (3.39) and (3.40) can be estimated in a similar manner. Combining the above estimates and using (3.37), (3.38) we obtain

$$
\begin{aligned}
& \left\|\mathcal{F}_{1}\left(\zeta^{1}, \bar{u}^{1}\right)-\mathcal{F}_{1}\left(\zeta^{2}, \bar{u}^{2}\right)\right\|_{L^{2}\left(Q_{0, T_{*}}\right)}+\left\|\mathcal{F}_{2}\left(\zeta^{1}, \bar{u}^{1}\right)-\mathcal{F}_{2}\left(\zeta^{2}, \bar{u}^{2}\right)\right\|_{L^{2}\left[0, T_{*}\right]} \\
& \leqslant K_{3} T_{*}^{1 / 8}\left(\|\breve{u}\|_{H^{2,1}\left(Q_{0, T_{*}}\right)}+\|\breve{\zeta}\|_{C\left(\left[0, T_{*}\right] ; H^{1}\left(\Omega_{0}\right)\right)}\right) \\
& \leqslant K_{3} T_{*}^{1 / 8}\left(\left\|f_{1}^{1}-f_{1}^{2}\right\|_{L^{2}\left(Q_{0, T_{*}}\right)}+\left\|f_{2}^{1}-f_{2}^{2}\right\|_{L^{2}\left[0, T_{*}\right]}\right)
\end{aligned}
$$

We are now in a position to prove the main result in this section:

Proof of Theorem 3.1. Fix $\varepsilon<\frac{1}{M}$. Let us define

$$
T_{M}=\min \left\{1, \widetilde{T}, \frac{1}{K_{2}^{8}}, \frac{1}{256 K_{3}^{8}}, \frac{1}{K_{1}^{2}}\left(\frac{1}{M}-\varepsilon\right)^{2}\right\},
$$

where $\widetilde{T}$ is the constant in Proposition 3.5 and $K_{1}, K_{2}, K_{3}$ are the constants in Proposition 3.3, Lemma 3.6 and Lemma 3.7, respectively. For $0<T_{*} \leqslant T_{M}$, we consider the map

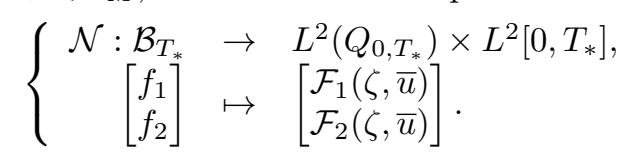

where $\mathcal{F}_{1}, \mathcal{F}_{2}$ have been defined in (3.6) and (3.7). To prove the claimed conclusions, it suffices to show that for every $T_{*} \in\left(0, T_{M}\right]$ the mapping $\mathcal{N}$ is a strict contraction of $\mathcal{B}_{T_{*}}$, with a Lipschitz constant $\frac{1}{2}$.

We first note that from Lemma 3.6 we obtain the existence of $K_{2}>0$, depending only on $M$, such that

$$
\left\|\mathcal{N}\left[\begin{array}{l}
f_{1} \\
f_{2}
\end{array}\right]\right\|_{L^{2}\left(Q_{0, T_{*}}\right) \times L^{2}\left[0, T_{*}\right]} \leqslant K_{2} T_{*}^{1 / 8} \quad\left(\left[\begin{array}{l}
f_{1} \\
f_{2}
\end{array}\right] \in \mathcal{B}_{T_{*}}\right) .
$$

From the definition of $T_{M}$ it is easy to see that $\mathcal{N}$ maps $\mathcal{B}_{T_{*}}$ into $\mathcal{B}_{T_{*}}$ for every $T \in\left(0, T_{M}\right]$. Next from Lemma 3.7 , there exists $K_{3}>0$, depending only on $M$, such that

$$
\begin{aligned}
\left\|\mathcal{N}\left[\begin{array}{l}
f_{1}^{1} \\
f_{2}^{1}
\end{array}\right]-\mathcal{N}\left[\begin{array}{l}
f_{1}^{2} \\
f_{2}^{2}
\end{array}\right]\right\|_{L^{2}\left(Q_{0, T_{*}}\right) \times L^{2}\left[0, T_{*}\right]} \leqslant K_{3} T_{*}^{1 / 8}\left(\left\|f_{1}^{1}-f_{1}^{2}\right\|_{L^{2}\left(Q_{0, T_{*}}\right)}+\left\|f_{2}^{1}-f_{2}^{2}\right\|_{L^{2}\left[0, T_{*}\right]}\right) \\
\\
\qquad\left(\left[\begin{array}{l}
f_{1}^{j} \\
f_{2}^{j}
\end{array}\right] \in \mathcal{B}_{T_{*}}, j=1,2\right) .
\end{aligned}
$$

Again from the definition of $T_{M}$ one can easily check that $\mathcal{N}$ is a strict contraction of $\mathcal{B}_{T_{*}}$ for every $T_{*} \in\left(0, T_{M}\right]$, which implies our existence and uniqueness result. Using (3.11) and definition of $T_{M}$ we have for every $t \in\left[0, T_{*}\right]$

$$
\left|h(t)-h_{0}\right| \leqslant T_{*}^{1 / 2}\|h\|_{H^{2}\left(0, T_{*}\right)} \leqslant\left(\frac{1}{M}-\varepsilon\right) .
$$

Therefore $h(t) \in[-1+\varepsilon, 1-\varepsilon]$, for all $t \in\left[0, T_{*}\right]$.

Finally, the fact that the Lipschitz constant does not depend on the initial and boundary data ensures the continuity of the map defined in (3.4) (see, for instance, Theorem 3.8 in [5]). 


\section{MAss AND ENERGy ESTimates}

Within this section and in the following one we assume that the initial and boundary data $\rho_{0}, u_{0}, h_{0}, \rho_{-1}$, $\rho_{1}, u_{-1}, u_{1}$ satisfy the assumptions in Theorem 3.1 and we use the notation from the above quoted theorem for the constant $M$. We assume that $T$ is any fixed number in $[1, \infty)$, that $\tau \in[0, T]$ and that $(h, \rho, u)$ is a strong solution solution of (1.1), (1.2) and (1.5) defined on $[0, \tilde{\tau}]$ for every $\tilde{\tau} \in(0, \tau)$. The results below adapt in an obvious manner for the local in time solutions of (1.1), (1.2), (1.3) and (1.1), (1.2), (1.4). In the remaining part of this section $C$ denotes a positive constant depending only on $M$ and $\tau$ that may change from line to line.

Our first result in this section says, roughly speaking, that the mass of gas on each side of the piston does not blow up in finite time.

Lemma 4.1. For every $t \in[0, \tau)$ we have

$$
\int_{-1}^{h(t)} \rho(t, x) \mathrm{d} x \leqslant C, \quad \int_{h(t)}^{1} \rho(t, x) \mathrm{d} x \leqslant C .
$$

Proof. Integrating (1.1a) on $[-1, h(t)]$, we obtain that for a.e. $t \in[0, \tau)$ we have

$$
\frac{\mathrm{d}}{\mathrm{d} t} \int_{-1}^{h(t)} \rho(t, x) \mathrm{d} x-\rho\left(t, h(t)^{-}\right) \dot{h}(t)+\rho\left(t, h(t)^{-}\right) u\left(t, h(t)^{-}\right)-\rho_{-1}(t) u_{-1}(t)=0 .
$$

Using (1.1c) we see that the second and the third term in the above formula cancel, so that, integrating with respect to time, we obtain that

$$
\int_{-1}^{h(t)} \rho(t, x) \mathrm{d} x=\int_{-1}^{h_{0}} \rho_{0}(x) \mathrm{d} x+\int_{0}^{t} \rho_{-1}(\sigma) u_{-1}(\sigma) \mathrm{d} \sigma \quad(t \in[0, \tau)) .
$$

Similarly, we obtain

$$
\int_{h(t)}^{1} \rho(t, x) \mathrm{d} x=\int_{h_{0}}^{1} \rho_{0}(x) \mathrm{d} x+\int_{0}^{t} \rho_{1}(\sigma) u_{1}(\sigma) \mathrm{d} \sigma \quad(t \in[0, \tau)) .
$$

The last two estimates, combined with (3.2), yield the desired result.

The next result is an energy estimate which plays an important role in the remaining part or the paper.

Proposition 4.2. There exists a strictly positive constant $C=C(M, \tau)$ such that

$$
\int_{-1}^{1} \rho(t, x) u^{2}(t, x) \mathrm{d} x+\int_{-1}^{1} \rho^{\gamma}(t, x) \mathrm{d} x+\dot{h}^{2}(t) \leqslant C \quad(t \in[0, \tau)) .
$$

Proof. For $t \in[0, \tau)$ and $x \in[-1,1]$ we set $g(t, x)=\frac{1-x}{2} u_{-1}(t)-\frac{1+x}{2} u_{1}(t)$ and we denote $w=u-g$. We note that

$$
\begin{array}{ccc}
w(t,-1)=0, \quad w(t, 1)=0 & (t \in[0, \tau)), & \\
\rho\left(\partial_{t} w+u \partial_{x} w+\partial_{t} g+u \partial_{x} g\right)-\partial_{x x} w+\partial_{x}\left(\rho^{\gamma}\right)=0 & (t \in[0, \tau), x \in(-1,1) \backslash\{h(t)\}, \\
w(t, h(t))=\dot{h}(t)-\frac{1-h(t)}{2} u_{-1}(t)+\frac{1+h(t)}{2} u_{1}(t) & (t \in[0, \tau)) .
\end{array}
$$

Moreover, since $g$ is continuous with respect to $x,(1.1 \mathrm{~d})$ yields that

$$
m \ddot{h}(t)=\left[\partial_{x} w-\rho^{\gamma}\right](t, h(t)) \quad(t \in[0, \tau)) .
$$

We next multiply (4.5) by $w$ and we integrate on $[-1, h(t)]$. Using (4.4), it is easily seen that the contributions of each term in the left hand side of (4.5) writes:

$$
\begin{gathered}
\int_{-1}^{h(t)} \rho\left(\partial_{t} w\right) w \mathrm{~d} x=\frac{1}{2} \frac{\mathrm{d}}{\mathrm{d} t} \int_{-1}^{h(t)} \rho w^{2} \mathrm{~d} x-\frac{1}{2} \int_{-1}^{h(t)}\left(\partial_{t} \rho\right) w^{2} \mathrm{~d} x-\frac{\dot{h}(t)}{2}\left(\rho w^{2}\right)\left(t, h(t)^{-}\right) \quad(t \in[0, \tau)), \\
\int_{-1}^{h(t)} \rho u\left(\partial_{x} w\right) w \mathrm{~d} x=\left(\rho u \frac{w^{2}}{2}\right)\left(t, h(t)^{-}\right)-\int_{-1}^{h(t)} \partial_{x}(\rho u) \frac{w^{2}}{2} \mathrm{~d} x \quad(t \in[0, \tau),
\end{gathered}
$$


Consequently, we obtain

$$
\begin{gathered}
\int_{-1}^{h(t)}\left(-\partial_{x x} w\right) w \mathrm{~d} x=-\left(\left(\partial_{x} w\right) w\right)\left(t, h(t)^{-}\right)+\int_{-1}^{h(t)}\left(\partial_{x} w\right)^{2} \mathrm{~d} x \quad(t \in[0, \tau)), \\
\int_{-1}^{h(t)}\left(\partial_{x} \rho^{\gamma}\right) w \mathrm{~d} x=\left(\rho^{\gamma} w\right)\left(t, h(t)^{-}\right)-\int_{-1}^{h(t)} \rho^{\gamma}\left(\partial_{x} w\right) \mathrm{d} x \quad(t \in[0, \tau)) .
\end{gathered}
$$

$$
\begin{aligned}
\frac{1}{2} \frac{\mathrm{d}}{\mathrm{d} t} \int_{-1}^{h(t)} \rho w^{2} \mathrm{~d} x & -\frac{1}{2} \int_{-1}^{h(t)}\left(\partial_{t} \rho+\partial_{x}(\rho u)\right) w^{2} \mathrm{~d} x+\frac{u\left(t, h(t)^{-}\right)-\dot{h}(t)}{2}\left(\rho w^{2}\right)\left(t, h(t)^{-}\right) \\
& -\left(\partial_{x} w-\rho^{\gamma}\right)\left(t, h(t)^{-}\right) w(t, h(t))+\int_{-1}^{h(t)}\left(\partial_{x} w\right)^{2} \mathrm{~d} x-\int_{-1}^{h(t)} \rho^{\gamma}\left(\partial_{x} w\right) \mathrm{d} x \\
& =-\int_{-1}^{h(t)} \rho\left(\partial_{t} g+u \partial_{x} g\right) w \mathrm{~d} x \quad(t \in[0, \tau))
\end{aligned}
$$

Combining the above relation with (1.1a) and (1.1c), we obtain

$$
\begin{aligned}
\frac{1}{2} \frac{\mathrm{d}}{\mathrm{d} t} \int_{-1}^{h(t)} \rho w^{2} \mathrm{~d} x-\left(\partial_{x} w-\rho^{\gamma}\right)\left(t, h(t)^{-}\right) w(t, h(t)) & +\int_{-1}^{h(t)}\left(\partial_{x} w\right)^{2} \mathrm{~d} x-\int_{-1}^{h(t)} \rho^{\gamma}\left(\partial_{x} w\right) \mathrm{d} x \\
& =-\int_{-1}^{h(t)} \rho\left(\partial_{t} g+u \partial_{x} g\right) w \mathrm{~d} x \quad(t \in[0, \tau))
\end{aligned}
$$

On the other hand, it is easily checked that for any smooth function $b: \mathbb{R}_{+}^{*} \rightarrow \mathbb{R}$, relation (1.1a) yields

$$
\partial_{t}(b(\rho))+\partial_{x}(b(\rho) u)=\left(b(\rho)-\rho b^{\prime}(\rho)\right) \partial_{x} u \quad(t \in[0, \tau), x \in[-1,1] \backslash\{h(t)\}) .
$$

In particular, if we set

$$
b(\rho)=\frac{\rho^{\gamma}}{\gamma-1} \quad \text { if } \quad \gamma>1, \quad b(\rho)=\rho \ln (\rho)-\rho \quad \text { if } \quad \gamma=1
$$

we deduce from (4.9) that

$$
\partial_{t}(b(\rho))+\partial_{x}(b(\rho) u)=-\rho^{\gamma} \partial_{x} u \quad(t \in[0, \tau), x \in[-1,1] \backslash\{h(t)\}) .
$$

The above relation and $(1.1 \mathrm{c}),(1.5)$ yield

$$
-\int_{-1}^{h(t)} \rho^{\gamma}\left(\partial_{x} u\right) \mathrm{d} x=\int_{-1}^{h(t)} \partial_{t}(b(\rho))+\partial_{x}(b(\rho) u) \mathrm{d} x=\frac{\mathrm{d}}{\mathrm{d} t} \int_{-1}^{h(t)} b(\rho) \mathrm{d} x-b\left(\rho_{-1}(t)\right) u_{-1}(t) \quad(t \in[0, \tau)) .
$$

Therefore, using the definitions of $g$ and of $w$ from the beginning of the proof, we have

$$
-\int_{-1}^{h(t)} \rho^{\gamma}\left(\partial_{x} w\right) \mathrm{d} x=\frac{\mathrm{d}}{\mathrm{d} t} \int_{-1}^{h(t)} b(\rho) \mathrm{d} x-b\left(\rho_{-1}(t)\right) u_{-1}(t)+\left(\frac{u_{1}(t)}{2}+\frac{u_{-1}(t)}{2}\right) \int_{-1}^{h(t)} \rho^{\gamma} \mathrm{d} x .
$$

Combining the above relation with (4.8) yields

$$
\begin{aligned}
& \frac{1}{2} \frac{\mathrm{d}}{\mathrm{d} t} \int_{-1}^{h(t)} \rho w^{2} \mathrm{~d} x+\frac{\mathrm{d}}{\mathrm{d} t} \int_{-1}^{h(t)} b(\rho) \mathrm{d} x-\left(\partial_{x} w-\rho^{\gamma}\right)\left(t, h(t)^{-}\right) w(t, h(t))+\int_{-1}^{h(t)}\left(\partial_{x} w\right)^{2} \mathrm{~d} x \\
& =b\left(\rho_{-1}(t)\right) u_{-1}(t)-\left(\frac{u_{1}(t)}{2}+\frac{u_{-1}(t)}{2}\right) \int_{-1}^{h(t)} \rho^{\gamma} \mathrm{d} x-\int_{-1}^{h(t)} \rho\left(\partial_{t} g+u \partial_{x} g\right) w \mathrm{~d} x \quad(t \in[0, \tau))
\end{aligned}
$$

By doing similar calculations for $x \in(h(t), 1)$ we obtain that

$$
\begin{aligned}
\frac{1}{2} \frac{\mathrm{d}}{\mathrm{d} t} \int_{h(t)}^{1} \rho w^{2} \mathrm{~d} x+\frac{\mathrm{d}}{\mathrm{d} t} \int_{h(t)}^{1} b(\rho) \mathrm{d} x+\left(\partial_{x} w-\rho^{\gamma}\right)\left(t, h(t)^{+}\right) w(t, h(t))+\int_{h(t)}^{1}\left(\partial_{x} w\right)^{2} \mathrm{~d} x \\
=-b\left(\rho_{1}(t)\right) u_{1}(t)-\left(\frac{u_{1}(t)}{2}+\frac{u_{-1}(t)}{2}\right) \int_{h(t)}^{1} \rho^{\gamma} \mathrm{d} x-\int_{h(t)}^{1} \rho\left(\partial_{t} g+u \partial_{x} g\right) w \mathrm{~d} x \quad(t \in[0, \tau))
\end{aligned}
$$


Adding the above relation and (4.11) and using (4.6), (4.7), we deduce that

$$
\begin{aligned}
& \frac{1}{2} \frac{\mathrm{d}}{\mathrm{d} t} \int_{-1}^{1} \rho w^{2} \mathrm{~d} x+\frac{\mathrm{d}}{\mathrm{d} t} \int_{-1}^{1} b(\rho) \mathrm{d} x+m \ddot{h}(t)\left(\dot{h}(t)-\frac{1-h(t)}{2} u_{-1}(t)-\frac{1+h(t)}{2} u_{1}(t)\right)+\int_{-1}^{1}\left(\partial_{x} w\right)^{2} \mathrm{~d} x \\
& =b\left(\rho_{-1}(t)\right) u_{-1}(t)-b\left(\rho_{1}(t)\right) u_{1}(t)+\left(\frac{u_{-1}(t)}{2}+\frac{u_{1}(t)}{2}\right) \int_{-1}^{1} \rho^{\gamma} \mathrm{d} x-\int_{-1}^{1} \rho\left(\partial_{t} g+u \partial_{x} g\right) w \mathrm{~d} x \quad(t \in[0, \tau)) .
\end{aligned}
$$

Integrating all the terms in the above formula with respect to time it follows that

$$
\begin{aligned}
& \frac{1}{2} \int_{-1}^{1} \rho w^{2} \mathrm{~d} x+\int_{-1}^{1} b(\rho) \mathrm{d} x+\frac{m}{2} \dot{h}^{2}(t)+\int_{0}^{t} \int_{-1}^{1}\left(\partial_{x} w\right)^{2} \mathrm{~d} x \\
& =\frac{1}{2} \int_{-1}^{1} \rho_{0}(x) w(0, x)^{2} \mathrm{~d} x+\int_{-1}^{1} b\left(\rho_{0}\right) \mathrm{d} x+\frac{m}{2} \ell_{0}^{2}+\int_{0}^{t}\left(b\left(\rho_{-1}(s)\right) u_{-1}(s)-b\left(\rho_{1}(s)\right) u_{1}(s)\right) \mathrm{d} s \\
& \quad+\frac{1}{2} \int_{0}^{t} \int_{-1}^{1} \rho^{\gamma}\left(u_{-1}(s)+u_{1}(s)\right) \mathrm{d} x \mathrm{~d} s-\int_{0}^{t} \int_{-1}^{1} \rho\left(\partial_{t} g+u \partial_{x} g\right) w \mathrm{~d} x \mathrm{~d} s \\
& \quad+\int_{0}^{t} m \ddot{h}\left(\frac{1-h}{2}\right) u_{-1} \mathrm{~d} s+\int_{0}^{t} m \ddot{h}\left(\frac{1+h}{2}\right) u_{1} \mathrm{~d} s \quad(t \in[0, \tau))
\end{aligned}
$$

We next estimate the last three terms in the right hand side of the above formula. For the first one, based on the definitions of $w$ and $g$ from the beginning of the proof, we have

$$
\int_{0}^{t} \int_{-1}^{1} \rho\left(\partial_{t} g+u \partial_{x} g\right) w \mathrm{~d} x \mathrm{~d} s=\int_{0}^{t} \int_{-1}^{1} \rho w\left(\partial_{t} g+(w+g) \partial_{x} g\right) \mathrm{d} x \mathrm{~d} s \quad(t \in[0, \tau))
$$

The first term in the right hand side of the above formula satisfies, with $C=C(M)$,

$$
\left|\int_{0}^{t} \int_{-1}^{1} \rho\left(\partial_{t} g+g \partial_{x} g\right) w \mathrm{~d} x \mathrm{~d} s\right| \leqslant C\left(\int_{0}^{t}\left(\left(\dot{u}_{-1}\right)^{2}+\left(\dot{u}_{1}\right)^{2}\right) \int_{-1}^{1} \rho \mathrm{d} x \mathrm{~d} s+\int_{0}^{t} \int_{-1}^{1} \rho w^{2} \mathrm{~d} x \mathrm{~d} s\right), \quad(t \in[0, \tau)) .
$$

By combining the above inequality and Lemma 4.1 it follows that

$$
\left|\int_{0}^{t} \int_{-1}^{1} \rho\left(\partial_{t} g+g \partial_{x} g\right) w \mathrm{~d} x \mathrm{~d} s\right| \leqslant C\left(1+\int_{0}^{t} \int_{-1}^{1} \rho w^{2} \mathrm{~d} x \mathrm{~d} s\right) \quad(t \in[0, \tau)) .
$$

To estimate the last two terms in the right hand side of (4.12) we note that integrating by parts we have

$$
\begin{aligned}
\int_{0}^{t} m \ddot{h}(s)\left(\frac{1-h(s)}{2}\right) u_{-1}(s) \mathrm{d} s=m \dot{h}(t)\left(\frac{1-h(t)}{2}\right) u_{-1}(t)-m \ell_{0}\left(\frac{1-h_{0}}{2}\right) u_{-1}(0) & \\
& \quad+m \int_{0}^{t} \frac{(\dot{h})^{2}(s)}{2}-\left(\frac{1-h(s)}{2}\right) \dot{h}(s) \dot{u}_{-1}(s) \mathrm{d} s \quad(t \in[0, \tau))
\end{aligned}
$$

Using the fact that $h(t)=h_{0}+\int_{0}^{t} \dot{h}(s) \mathrm{d} s$, together with (3.2), (3.3), we deduce that

$$
\left|\int_{0}^{t} m \ddot{h}(s)\left(\frac{1-h(s)}{2}\right) u_{-1}(s) \mathrm{d} s\right| \leqslant m \frac{\dot{h}^{2}(t)}{8}+C\left(1+\int_{0}^{t} \dot{h}^{2}(s) \mathrm{d} s\right) \quad(t \in[0, \tau) .
$$

Similarly, we obtain

$$
\left|\int_{0}^{t} m \ddot{h}(s)\left(\frac{1+h(s)}{2}\right) u_{1}(s) \mathrm{d} s\right| \leqslant m \frac{\dot{h}^{2}(t)}{8}+C\left(1+\int_{0}^{t} \dot{h}^{2}(s) \mathrm{d} s\right) \quad(t \in[0, \tau)) .
$$


To estimate the forth and fifth terms in the right hand side of (4.12) we note that, since $b \in C^{1}\left(\mathbb{R}_{+}^{*}\right)$, we can use (3.2) to obtain

$$
\begin{aligned}
\int_{0}^{t}\left(b\left(\rho_{-1}(s)\right) u_{-1}(s)-b\left(\rho_{1}(s)\right) u_{1}(s)\right) \mathrm{d} s+\frac{1}{2} \int_{0}^{t} \int_{-1}^{1} \rho^{\gamma}\left(u_{-1}(s)+u_{1}(s)\right) \mathrm{d} x \mathrm{~d} s & \leqslant C\left(1+\int_{0}^{t} \int_{-1}^{1} \rho^{\gamma} \mathrm{d} x \mathrm{~d} s\right) \quad(t \in[0, \tau)) .
\end{aligned}
$$

Gathering (4.12), (4.13), (4.14), (4.15) and (4.16), we conclude that there exists $C=C(\tau, M)>0$ such that for every $t \in[0, \tau)$ we have

$$
\int_{-1}^{1} \rho w^{2} \mathrm{~d} x+\int_{-1}^{1} b(\rho) \mathrm{d} x+\dot{h}^{2} \leqslant C\left(1+\int_{0}^{t} \int_{-1}^{1} \rho w^{2} \mathrm{~d} x \mathrm{~d} s+\int_{0}^{t} \int_{-1}^{1} \rho^{\gamma} \mathrm{d} x \mathrm{~d} s+\int_{0}^{t} \dot{h}^{2}(s) \mathrm{d} s\right) .
$$

Finally, using Gronwall's Lemma, we deduce that the conclusion (4.3) holds for $\gamma>1$.

In the case $\gamma=1$, we notice that (4.17) and Lemma 4.1 yield

$$
\int_{-1}^{1} \rho w^{2} \mathrm{~d} x+\dot{h}^{2} \leqslant C\left(1+\int_{0}^{t} \int_{-1}^{1} \rho w^{2} \mathrm{~d} x \mathrm{~d} s+\int_{0}^{t} \dot{h}^{2}(s) \mathrm{d} s\right) .
$$

We can then conclude as in the case $\gamma>1$.

\section{Estimates ON THE DENSITy FIELD}

Within this section we continue to use the assumptions in Section 4, which means that $(h, \rho, u)$ is a strong solution solution of (1.1), (1.2) and (1.5) defined on $[0, \tilde{\tau}]$ for every $\tilde{\tau} \in[0, \tau)$, with the initial and boundary data $\rho_{0}, u_{0}, h_{0}, \rho_{-1}, \rho_{1}, u_{-1}, u_{1}$ and the constant $M$ satisfying the assumptions in Theorem 3.1. We continue to derive estimates of this strong solution, focusing on pointwise estimates for the density and on estimates of the derivatives of $u$ in Lebesgue-Sobolev spaces.

Let $Q_{h, \tau}$ be the set which has been defined in (2.4)) and let $B: Q_{h, \tau} \rightarrow \mathbb{R}$ be defined by

$$
\begin{array}{ll}
B(t, x)=\int_{h(t)}^{x} \rho(t, y) u(t, y) \mathrm{d} y+\int_{0}^{t}\left(\partial_{x} u-\rho^{\gamma}\right)\left(s, h(s)^{+}\right) \mathrm{d} s & (x>h(t)), \\
B(t, x)=\int_{h(t)}^{x} \rho(t, y) u(t, y) \mathrm{d} y-\int_{0}^{t}\left(\partial_{x} u-\rho^{\gamma}\right)\left(s, h(s)^{-}\right) \mathrm{d} s & (x<h(t)) .
\end{array}
$$

The above assumptions imply that, $B \in H^{1}\left(Q_{h, \widetilde{\tau}}^{+}\right) \cap H^{1}\left(Q_{h, \widetilde{\tau}}^{-}\right)$for every $\widetilde{\tau} \in[0, \tau)$, where $Q_{h, \widetilde{\tau}}^{+}$and $Q_{h, \widetilde{\tau}}^{-}$are defined by

$$
\begin{aligned}
& Q_{h, \widetilde{\tau}}^{+}:=\{(t, x) \in(0, \widetilde{\tau}) \times(-1,1) ; x>h(t)\}, \\
& Q_{h, \widetilde{\tau}}^{-}:=\{(t, x) \in(0, \widetilde{\tau}) \times(-1,1) ; x<h(t)\} .
\end{aligned}
$$

Lemma 5.1. The function $B$ satisfies, for every $\widetilde{\tau} \in[0, \tau)$,

$$
\begin{array}{crr}
\partial_{x} B=\rho u, \quad \partial_{t} B=\partial_{x} u-\rho^{\gamma}-\rho u^{2} & \text { in } & \mathcal{D}^{\prime}\left(Q_{h, \widetilde{\tau}}^{-}\right) \cap \mathcal{D}^{\prime}\left(Q_{h, \widetilde{\tau}}^{+}\right), \\
B(0, x):=B_{0}(x)=\int_{h_{0}}^{x} \rho_{0}(y) u_{0}(y) \mathrm{d} y & (x \in[-1,1]) .
\end{array}
$$

Proof. We prove only the second relation, the two others following directly from the definition of $B$. To this aim, we first note that for $t \in[0, \tau)$ and $x>h(t)$ we have

$$
\partial_{t}\left(\int_{h(t)}^{x} \rho(t, y) u(t, y) \mathrm{d} y\right)=-\rho\left(t, h(t)^{+}\right) u\left(t, h(t)^{+}\right) \dot{h}(t)+\int_{h(t)}^{x}\left[\left(\partial_{t} \rho\right) u+\rho\left(\partial_{t} u\right)\right] \mathrm{d} y .
$$


Combining the above equation with (1.1a) and (1.1b), we deduce

$$
\begin{aligned}
\partial_{t}\left(\int_{h(t)}^{x} \rho(t, y) u(t, y) \mathrm{d} y\right)=-\rho\left(t, h(t)^{+}\right) u\left(t, h(t)^{+}\right) \dot{h}(t) & +\int_{h(t)}^{x} \partial_{x}\left(-\rho u^{2}+\partial_{x} u-\rho^{\gamma}\right) \mathrm{d} y \\
& =\left(-\rho u^{2}+\partial_{x} u-\rho^{\gamma}\right)(t, x)-\left(\partial_{x} u-\rho^{\gamma}\right)\left(t, h(t)^{+}\right),
\end{aligned}
$$

i.e. that (5.3) holds in $\mathcal{D}^{\prime}\left(Q_{h, \widetilde{\tau}}^{+}\right)$for every $\widetilde{\tau} \in[0, \tau)$.

A similar calculation can be done for $x<h(t)$, leading to the fact that (5.3) holds in $\mathcal{D}^{\prime}\left(Q_{h, \widetilde{\tau}}^{-}\right)$for every $\widetilde{\tau} \in[0, \tau)$.

Combining (5.3) and (1.1a), it is not difficult check that for every $\widetilde{\tau} \in[0, \tau)$,

$$
\begin{aligned}
& \partial_{t}(B+\ln \rho)+u \partial_{x}(B+\ln \rho)+\rho^{\gamma}=0 \text { in } \mathcal{D}^{\prime}\left(Q_{h, \tilde{\tau}}^{-}\right) \cap \mathcal{D}^{\prime}\left(Q_{h, \widetilde{\tau}}^{+}\right), \\
& \partial_{t}\left(\rho e^{B}\right)+u \partial_{x}\left(\rho e^{B}\right)+\rho^{\gamma+1} e^{B}=0 \text { in } \mathcal{D}^{\prime}\left(Q_{h, \tilde{\tau}}^{-}\right) \cap \mathcal{D}^{\prime}\left(Q_{h, \tau}^{+}\right), \\
& \partial_{t}\left(\frac{1}{\rho} e^{-B}\right)+u \partial_{x}\left(\frac{1}{\rho} e^{-B}\right)-\rho^{\gamma-1} e^{-B}=0 \quad \text { in } \quad \mathcal{D}^{\prime}\left(Q_{h, \tilde{\tau}}^{-}\right) \cap \mathcal{D}^{\prime}\left(Q_{h, \tilde{\tau}}^{+}\right) .
\end{aligned}
$$

For a detailed proof and more discussion of the above formulas we refer to [20], [12]. The function $B$ introduced above is an adaptation of a similar function introduced in [20] and then discussed in detail in [12]. However, unlike in the above mentioned references, the function $t \mapsto B(t, \cdot)$ is not continuous (it has a jump at $x=h(t)$ ). Since the continuity of $B$ plays an important role in the above quoted papers, we first modify $B$ to get a continuous function $\tilde{B}$. More precisely, we set:

$$
\widetilde{B}(t, x)=\left\{\begin{array}{l}
B(t, x)-\frac{m}{2}\left(\dot{h}(t)-\ell_{0}\right) \text { for } x \in(-1, h(t)), \\
B(t, x)+\frac{m}{2}\left(\dot{h}(t)-\ell_{0}\right) \text { for } x \in(h(t), 1) .
\end{array}\right.
$$

Lemma 5.2. Let $B$ and $\widetilde{B}$ be defined by (5.1), (5.2), and (5.8). Then for every $\tilde{\tau} \in[0, \tau]$ we have

$$
\widetilde{B} \in H^{1}\left((0, \widetilde{\tau}) ; L^{2}[-1,1]\right) \cap L^{2}\left([0, \widetilde{\tau}] ; H^{1}(-1,1)\right) .
$$

Proof. Since we have the desired properties on each side of $h(t)$, we only need to show that

$$
\widetilde{B}\left(t, h(t)^{-}\right)=\widetilde{B}\left(t, h(t)^{+}\right) .
$$

From (5.1) and (5.2), we deduce

$$
[B](t, h(t))=\int_{0}^{t} m \ddot{h} \mathrm{~d} s=m\left(\dot{h}(t)-\ell_{0}\right) .
$$

Thus $[\widetilde{B}](t, h(t))=0$ which gives the result.

Lemma 5.3. There exists a positive constant $C>0$, depending only on $M$ and on $\tau$, such that for every $t \in[0, \tau)$ we have

$$
\sup _{x \in[-1,1] \backslash\{h(t)\}}|B| \leqslant C\left(1+\left(\int_{-1}^{1} \rho(t, x) u^{2}(t, x) \mathrm{d} x\right)^{1 / 2}+\int_{0}^{t} \int_{-1}^{1}\left(\rho^{\gamma}(\sigma, x)+\rho(\sigma, x) u^{2}(\sigma, x)\right) \mathrm{d} x \mathrm{~d} \sigma\right) .
$$

Proof. Using (5.9) we can write

$$
\begin{aligned}
& \int_{-1}^{1} \widetilde{B}(t, x) \mathrm{d} x=\int_{0}^{t} \int_{-1}^{1} \partial_{t} \widetilde{B}(\sigma, x) \mathrm{d} x \mathrm{~d} \sigma+\int_{-1}^{1} \widetilde{B}_{0}(x) \mathrm{d} x \\
& =\int_{0}^{t} \int_{-1}^{h(\sigma)}\left(\partial_{t} B(\sigma, x)-\frac{m}{2} \ddot{h}(\sigma)\right) \mathrm{d} x \mathrm{~d} \sigma+\int_{0}^{t} \int_{h(\sigma)}^{1}\left(\partial_{t} B+\frac{m}{2} \ddot{h}(\sigma)\right) \mathrm{d} x \mathrm{~d} \tau+\int_{-1}^{1} B_{0}(x) \mathrm{d} x \quad(t \in[0, \tau)) .
\end{aligned}
$$


Combining the above relation with (5.3) and (1.5), we obtain

$$
\begin{aligned}
& \int_{-1}^{1} \widetilde{B}(t, x) \mathrm{d} x=\int_{0}^{t} u_{1}(\sigma) \mathrm{d} \sigma-\int_{0}^{t} u_{-1}(\sigma) \mathrm{d} \sigma-\int_{0}^{t} \int_{-1}^{1}\left(\rho^{\gamma}(\sigma, x)+\rho(\sigma, x) u^{2}(\sigma, x)\right) \mathrm{d} x \mathrm{~d} \sigma \\
&+\int_{0}^{t} \frac{m}{2}(-(h(\sigma)+1) \ddot{h}(\sigma)+(1-h(\sigma)) \ddot{h}(\sigma)) \mathrm{d} \sigma+\int_{-1}^{1} B_{0}(x) \mathrm{d} x \quad(t \in[0, \tau)) .
\end{aligned}
$$

The above equality yields

$$
\begin{aligned}
\int_{-1}^{1} \widetilde{B}(t, x) \mathrm{d} x=\int_{0}^{t} u_{1}(\sigma) \mathrm{d} \sigma- & \int_{0}^{t} u_{-1}(\sigma) \mathrm{d} \sigma-\int_{0}^{t} \int_{-1}^{1}\left(\rho^{\gamma}(\sigma, x)+\rho(\sigma, x) u^{2}(\sigma, x)\right) \mathrm{d} x \mathrm{~d} \sigma \\
& +m \ell_{0} h_{0}-m \dot{h}(t) h(t)+m \int_{0}^{t} \dot{h}^{2}(\sigma) \mathrm{d} \sigma+\int_{-1}^{1} B_{0}(x) \mathrm{d} x \quad(t \in[0, \tau))
\end{aligned}
$$

Combining the above formula and Proposition 4.2, we deduce here exists a strictly positive constant $C=C(M, \tau)$ such that

$$
\left|\int_{-1}^{1} \widetilde{B}(t, x) \mathrm{d} x\right| \leqslant C+\int_{0}^{t} \int_{-1}^{1} \rho^{\gamma}(\sigma, x) \mathrm{d} x \mathrm{~d} \sigma \quad(t \in[0, \tau)) .
$$

On the other hand, using the regularity property (5.9) we have

$$
|\widetilde{B}(t, x)| \leqslant\left|\int_{-1}^{1} \widetilde{B}(t, y) \mathrm{d} y\right|+\int_{-1}^{1}\left|\partial_{x} \widetilde{B}(t, y)\right| \mathrm{d} y \quad(t \in[0, \tau)) .
$$

Combining the above estimate with (5.12) and (5.3), we obtain

$$
|\widetilde{B}(t, x)| \leqslant C+\int_{0}^{t} \int_{-1}^{1} \rho^{\gamma}(\sigma, x) \mathrm{d} x \mathrm{~d} \sigma+\left(\int_{-1}^{1} \rho(t, x) \mathrm{d} x\right)^{1 / 2}\left(\int_{-1}^{1} \rho(t, x) u^{2}(t, x) \mathrm{d} x\right)^{1 / 2} \quad(t \in[0, \tau)) .
$$

Gathering the above inequality, (5.8) and Proposition 4.2 completes the proof.

Lemma 5.4. For every $t \in[0, \tau)$ we have

$$
\begin{gathered}
\sup _{-1 \leqslant x<h(t)} \rho(t, x) \leqslant M \exp \left(2 \sup _{\substack{\sigma \in[0, \tau) \\
-1 \leqslant x<h(\sigma)}}|B(\sigma, x)|\right), \\
\sup _{h(t)<x \leqslant 1} \rho(t, x) \leqslant M \exp \left(2 \sup _{\substack{\sigma \in[0, \tau) \\
h(\sigma)<x \leqslant 1}}|B(\sigma, x)|\right),
\end{gathered}
$$

where $M$ is the constant introduced in Theorem 3.1 .

Proof. Multiplying (5.6) by $\rho\left(\rho e^{B}\right)^{n-1}$, integrating with respect to $x$ on $[-1, h(t)]$, and using (1.1a), (1.1c), we obtain

$$
\frac{1}{n} \frac{\mathrm{d}}{\mathrm{d} t} \int_{-1}^{h(t)} \rho\left(\rho e^{B}\right)^{n} \mathrm{~d} x-\frac{1}{n} \rho_{-1}(t) u_{-1}(t)\left[\rho_{-1}(t) e^{B(t,-1)}\right]^{n}+\int_{-1}^{h(t)} \rho^{\gamma+n+2} e^{n B} \mathrm{~d} x=0 \quad(t \in[0, \tau)) .
$$

Integrating the above formula with respect to time it follows that

$$
\begin{aligned}
\int_{-1}^{h(t)} \rho^{n+1} e^{n B} \mathrm{~d} x & \leqslant \int_{-1}^{h_{0}} \rho_{0}(x)^{n+1} e^{n B(0, x)} \mathrm{d} x+\int_{0}^{t} \rho_{-1}^{n+1}(\sigma) u_{-1}(\sigma) e^{n B(\sigma,-1)} \mathrm{d} \sigma \\
& \leqslant M^{n+1}\left(1+h_{0}+M \tau\right)\left[\sup _{\substack{\sigma \in[0, \tau) \\
-1 \leqslant x<h(\sigma)}} e^{B(\sigma, x)}\right]^{n} \quad(t \in[0, \tau)) .
\end{aligned}
$$


Thus

$$
\int_{-1}^{h(t)} \rho^{n+1} \mathrm{~d} x \leqslant M^{n+1}\left(1+h_{0}+M \tau\right)\left(\exp \left\{\sup _{\substack{\sigma \in[0, \tau) \\-1 \leqslant x<h(\sigma)}}|B(\sigma, x)|\right\}\right)^{n} \quad(t \in[0, \tau)) .
$$

Hence, for every $t \in[0, \tau)$ we have

$$
\sup _{-1 \leqslant x<h(t)} \rho(t, x)=\lim _{n \rightarrow \infty}\left(\int_{-1}^{h(t)} \rho^{n+1} \mathrm{~d} x\right)^{1 /(n+1)} \leqslant M \exp \left(2 \sup _{\substack{\sigma \in[0, \tau) \\-1 \leqslant x<h(\sigma)}}|B(\sigma, x)|\right),
$$

i.e., we have obtained the first inequality in the statement.

The proof of the second inequality, based on estimates for $x \in(h(t), 1)$, is completely similar.

Gathering Lemma 5.4, Lemma 5.3 and Proposition 4.2, we deduce the following result. (To be more precise, estimate (5.15) is a consequence of relations (4.1) and (4.2).).

Corollary 5.5. There exists a constant $C=C(\tau, M)>0$, depending on boundary such that

$$
\sup _{\substack{t \in[0, \tau) \\ x \in[-1,1] \backslash\{h(t)\}}} \rho(t, x) \leqslant C .
$$

Moreover,

$$
-1+\frac{1}{C} \int_{-1}^{h_{0}} \rho_{0}(x) \mathrm{d} x \leqslant h(t) \leqslant 1-\frac{1}{C} \int_{h_{0}}^{1} \rho_{0}(x) \mathrm{d} x \quad(t \in[0, \tau)) .
$$

The following generalization of Gronwall's lemma (we refer to [12] for its proof) is used below in proving Lemma 5.7.

Lemma 5.6. Assume $f \in C([0, T]), c \in L^{1}[0, T], f, c \geqslant 0$ and $a, b \in \mathbb{R}_{+}$. If

$$
f^{n}(t) \leqslant a+b \int_{0}^{t} c(s) f^{n-1}(s) \mathrm{d} s \quad(t \in[0, T])
$$

then

$$
f(t) \leqslant a^{1 / n}+\frac{b}{n} \int_{0}^{t} c(s) \mathrm{d} s \quad(t \in[0, T]) .
$$

The result below is an essential step in showing that the density field is bounded from below on bounded time intervals, i.e., that cavitation does not occur in finite time.

Lemma 5.7. For every $t \in[0, \tau)$ we have

$$
\begin{aligned}
& \sup _{-1 \leqslant x \leqslant h(t)} \frac{1}{\rho(t, x)} \leqslant M \exp \left(2 \sup _{\substack{\sigma \in[0, \tau) \\
-1 \leqslant x<h(\sigma)}}|B(\sigma, x)|\right)+C \exp \left(4 \sup _{\substack{\sigma \in[0, \tau) \\
-1 \leqslant x<h(\sigma)}}|B(\sigma, x)|\right) \\
& \sup _{h(t) \leqslant x \leqslant 1} \frac{1}{\rho(t, x)} \leqslant M \exp \left(2 \sup _{\substack{\sigma \in[0, \tau) \\
h(\sigma)<x \leqslant h(\sigma)}}|B(\sigma, x)|\right)+C \exp \left(\sup _{\substack{\sigma \in[0, \tau) \\
h(\sigma)<x \leqslant h(\sigma)}}|B(\sigma, x)|\right),
\end{aligned}
$$

where $M$ and $M_{1}$ are the constants introduced in Theorem 3.1 and in Corollary 5.5, respectively.

Proof. Multiplying (5.7) by $\rho\left(\rho e^{B}\right)^{-n}$, integrating with respect to $x$ in $[-1, h(t)]$, and using (1.1a), (1.1c), we obtain that for every $t \in[0, \tau)$ we have

$$
\frac{\mathrm{d}}{\mathrm{d} t} \int_{-1}^{h(t)} \rho\left(\frac{1}{\rho(t, x) e^{B(t, x)}}\right)^{n+1} \mathrm{~d} x=\rho_{-1}(t) u_{-1}(t)\left(\frac{1}{\rho_{-1}(t) e^{B(t,-1)}}\right)^{n+1}+(n+1) \int_{-1}^{h(t)} \rho^{\gamma-n}(t, x) e^{-(n+1) B} \mathrm{~d} x
$$


Integrating the above formula with respect to time we obtain, for every $t \in[0, \tau)$,

$$
\begin{aligned}
& \int_{-1}^{h(t)}\left(\frac{1}{\rho(t, x) e^{B(t, x)}}\right)^{n} e^{-B(t, x)} \mathrm{d} x=\int_{-1}^{h_{0}}\left(\frac{1}{\rho_{0}(x) e^{B(0, x)}}\right)^{n} e^{-B(0, x)} \mathrm{d} x \\
& +\int_{0}^{t} u_{-1}(s)\left(\frac{1}{\rho_{-1}(s) e^{B(s,-1)}}\right)^{n} e^{-B(s,-1)} \mathrm{d} s+(n+1) \int_{0}^{t} \int_{-1}^{h(s)} \rho^{\gamma-1} e^{-2 B(s, x)}\left(\frac{1}{\rho(s, x) e^{B(s, x)}}\right)^{n-1} \mathrm{~d} x \mathrm{~d} s
\end{aligned}
$$

Thus, using (3.2) and (3.3), we deduce that for every $t \in[0, \tau)$ we have

$$
\begin{array}{r}
\int_{-1}^{h(t)}\left(\frac{1}{\rho(t, x) e^{B(t, x)}}\right)^{n} e^{-B(t, x)} \mathrm{d} x \leqslant\left(1+h_{0}+M \tau\right) M^{n} \exp \left[(n+1) \sup _{\substack{\sigma \in[0, \tau) \\
-1 \leqslant x<h(\sigma)}}|B(\sigma, x)|\right] \\
+(n+1) \exp \left[2 \sup _{\substack{\sigma \in[0, \tau) \\
-1 \leqslant x<h(\sigma)}}|B(\sigma, x)|\right] \int_{0}^{t} \int_{-1}^{h(s)} \rho^{\gamma-1}\left(\frac{1}{\rho(s, x) e^{B(s, x)}}\right)^{n-1} \mathrm{~d} x \mathrm{~d} s
\end{array}
$$

The above estimates yields, for every $t \in[0, \tau)$,

$$
\begin{gathered}
\int_{-1}^{h(t)}\left(\frac{1}{\rho(t, x) e^{B(t, x)}}\right)^{n} \mathrm{~d} x \leqslant\left(1+h_{0}+M \tau\right) M^{n} \exp \left[(n+2) \sup _{\substack{\sigma \in[0, \tau) \\
-1 \leqslant x<h(\sigma)}}|B(\sigma, x)|\right] \\
+(n+1) \exp \left[3 \sup _{\substack{\sigma \in[0, \tau) \\
-1 \leqslant x<h(\sigma)}}|B(\sigma, x)|\right] \int_{0}^{t} \sup _{-1 \leqslant x \leqslant h(s)} \rho^{\gamma-1} \int_{-1}^{h(s)}\left(\frac{1}{\rho(s, x) e^{B(s, x)}}\right)^{n-1} \mathrm{~d} x \mathrm{~d} s
\end{gathered}
$$

We consider the continuous and positive function defined by

$$
f(t)=\left(\int_{-1}^{h(t)}\left(\frac{1}{\rho(t, x) e^{B(t, x)}}\right)^{n} \mathrm{~d} x\right)^{1 / n} \quad(t \in[0, \tau)) .
$$

Using the Hölder inequality, we have

$$
\int_{-1}^{h(t)}\left(\frac{1}{\rho e^{B(t, x)}}\right)^{n-1} \mathrm{~d} x \leqslant(h(t)+1)^{1 / n} f^{n-1}(t) \leqslant 2^{1 / n} f^{n-1}(t) \quad(t \in[0, \tau)) .
$$

Gathering the above equation, (5.17), Corollary 5.5, Proposition 4.2 and Lemma 5.3, we deduce

$$
\begin{array}{r}
f^{n}(t) \leqslant\left(1+h_{0}+M \tau\right) M^{n} \exp \left[(n+2) \sup _{\substack{\sigma \in[0, \tau) \\
-1 \leqslant x<h(\sigma)}}|B(\sigma, x)|\right] \\
+C 2^{1 / n}(n+1) \exp \left[3 \sup _{\substack{\sigma \in[0, \tau) \\
-1 \leqslant x<h(\sigma)}}|B(\sigma, x)|\right] \int_{0}^{t} f^{n-1}(s) \mathrm{d} s
\end{array}
$$


Applying Lemma 5.6 to the above estimate we obtain for all $n \geqslant 2$

$$
\begin{aligned}
\left(\int_{-1}^{h(t)}\left(\frac{1}{\rho(t, x) e^{B(t, x)}}\right)^{n} d x\right)^{1 / n} & \leqslant\left(1+h_{0}+M \tau\right)^{1 / n} M \exp \left[\left(1+\frac{2}{n}\right) \sup _{\substack{\sigma \in[0, \tau) \\
-1 \leqslant x<h(\sigma)}}|B(\sigma, x)|\right] \\
& +C 2^{1 / n} \tau\left(1+\frac{1}{n}\right) \exp \left[3 \sup _{\substack{\sigma \in[0, \tau) \\
-1 \leqslant x<h(\sigma)}}|B(\sigma, x)|\right] \quad(t \in[0, \tau))
\end{aligned}
$$

Therefore, for every $t \in[0, \tau)$, we have

$$
\begin{aligned}
\sup _{-1 \leqslant x<h(t)} \frac{1}{\rho(t, x) e^{B(t, x)}} & =\lim _{n \rightarrow \infty}\left(\int_{-1}^{h(t)}\left(\frac{1}{\rho(t, x) e^{B(t, x)}}\right)^{n} \mathrm{~d} x\right)^{1 / n} \\
& \leqslant M \exp \left[\sup _{\substack{\sigma \in[0, \tau) \\
-1 \leqslant x<h(\sigma)}}|B(\sigma, x)|\right]+C \tau \exp \left[3 \sup _{\substack{\sigma \in[0, \tau) \\
-1 \leqslant x<h(\sigma)}}|B(\sigma, x)|\right] .
\end{aligned}
$$

This gives the first inequality in the statement. The proof is similar when $x \in(h(t), 1)$.

Combining Lemma 5.7, Lemma 5.3 and Proposition 4.2, we deduce the following result.

Corollary 5.8. There exists a constant $C=C(M, \tau)$ such that

$$
\inf _{\substack{t \in[0, \tau) \\ x \in[-1,1] \backslash\{h(t)\}}} \rho(t, x) \geqslant C .
$$

\section{Proof of Theorem 1.1}

We first note that by combining (5.7) and Proposition 4.2 we obtain:

Lemma 6.1. There exists a constant $C=C(M, \tau)$ such that

$$
\|u(t, \cdot)\|_{L^{2}[-1,1]} \leqslant C \quad(t \in[0, \tau)) .
$$

We can also estimate the derivatives of $u$, as shown in the result below. Before that we state an elementary lemma.

Lemma 6.2. Assume $(a, b)$ is a nonempty bounded interval and $f \in H^{1}(a, b)$. Then for all $\varepsilon>0$ and $x \in(a, b)$,

$$
|f(x)|^{2} \leqslant\left(\frac{1}{b-a}+\frac{1}{\varepsilon}\right) \int_{a}^{b}|f(y)|^{2} \mathrm{~d} y+\varepsilon \int_{a}^{b}\left|f^{\prime}(y)\right|^{2} \mathrm{~d} y .
$$

Proof. It is sufficient to write for any $x, y \in(a, b)$

$$
|f(x)|^{2} \leqslant|f(y)|^{2}+\int_{a}^{b} 2|f(s)|\left|f^{\prime}(s)\right| \mathrm{d} s .
$$

Then we integrate in $y \in(a, b)$ and use Young's inequality.

Lemma 6.3. For every $t \in[0, \tau)$ the following estimates hold:

$$
\begin{gathered}
\int_{-1}^{h(t)}\left(\partial_{x} u\right)^{2} \mathrm{~d} x+\int_{0}^{t} \int_{-1}^{h(s)}\left[\left(\partial_{t} u\right)^{2}+\left(\partial_{x x} u\right)^{2}\right] \mathrm{d} x \mathrm{~d} s \leqslant C\left(1+\int_{0}^{t} \int_{-1}^{h(s)}\left(\partial_{x} \rho\right)^{2} \mathrm{~d} x \mathrm{~d} s\right), \\
\int_{h(t)}^{1}\left(\partial_{x} u\right)^{2} \mathrm{~d} x+\int_{0}^{t} \int_{h(s)}^{1}\left[\left(\partial_{t} u\right)^{2}+\left(\partial_{x x} u\right)^{2}\right] \mathrm{d} x \mathrm{~d} s \leqslant C\left(1+\int_{0}^{t} \int_{h(s)}^{1}\left(\partial_{x} \rho\right)^{2} \mathrm{~d} x \mathrm{~d} s\right),
\end{gathered}
$$

where $C=C(M, \tau)>0$. 
Proof. We first note that (1.1b) can be rewritten

$$
\rho^{1 / 2} \partial_{t} u-\rho^{-1 / 2} \partial_{x x} u=-\rho^{1 / 2} u \partial_{x} u-\gamma \rho^{\gamma-3 / 2} \partial_{x} \rho,
$$

so that for every $t \in[0, \tau)$ we have

$$
\int_{-1}^{h(t)}\left[\rho\left(\partial_{t} u\right)^{2}+\rho^{-1}\left(\partial_{x x} u\right)^{2}-2 \partial_{t} u \partial_{x x} u\right] \mathrm{d} x=\int_{-1}^{h(t)}\left(\rho^{1 / 2} u \partial_{x} u+\gamma \rho^{\gamma-3 / 2} \partial_{x} \rho\right)^{2} \mathrm{~d} x .
$$

Moreover, a simple calculation shows that

$$
\frac{\mathrm{d}}{\mathrm{d} t} \int_{-1}^{h(t)}\left(\partial_{x} u\right)^{2} \mathrm{~d} x=\left(\partial_{x} u\left(t, h(t)^{-}\right)^{2} \dot{h}(t)+2\left[\left(\partial_{x} u\right)\left(\partial_{t} u\right)\right]\left(t, h(t)^{-}\right)-\int_{-1}^{h(t)} 2\left(\partial_{x x} u\right)\left(\partial_{t} u\right) \mathrm{d} x \quad(t \in[0, \tau))\right.
$$

On the other hand, differentiating (1.1c) with respect to time we obtain

$$
\left(\partial_{t} u\right)\left(t, h(t)^{-}\right)+\dot{h}(t)\left(\partial_{x} u\right)\left(t, h(t)^{-}\right)=\ddot{h}(t) .
$$

Combining (6.3) and (6.4) yields

$$
-\int_{-1}^{h(t)} 2\left(\partial_{x x} u\right)\left(\partial_{t} u\right) \mathrm{d} x=\frac{\mathrm{d}}{\mathrm{d} t} \int_{-1}^{h(t)}\left(\partial_{x} u\right)^{2} \mathrm{~d} x+\left(\partial_{x} u\left(t, h(t)^{-}\right)^{2} \dot{h}(t)-2 \ddot{h}(t)\left(\partial_{x} u\right)\left(t, h(t)^{-}\right) \quad(t \in[0, \tau)) .\right.
$$

The above relation and (6.2) imply that for every $t \in[0, \tau)$ we have

$$
\begin{aligned}
& \int_{-1}^{h(t)}\left[\rho\left(\partial_{t} u\right)^{2}+\rho^{-1}\left(\partial_{x x} u\right)^{2}\right] \mathrm{d} x+\frac{\mathrm{d}}{\mathrm{d} t} \int_{-1}^{h(t)}\left(\partial_{x} u\right)^{2} \mathrm{~d} x \\
& \quad \leqslant 2 \int_{-1}^{h(t)}\left[\left(\rho^{1 / 2} u \partial_{x} u\right)^{2}+\left(\gamma \rho^{\gamma-3 / 2} \partial_{x} \rho\right)^{2}\right] \mathrm{d} x+\left(\partial_{x} u\left(t, h(t)^{-}\right)^{2}|\dot{h}(t)|+2|\ddot{h}(t)|\left|\left(\partial_{x} u\right)\left(t, h(t)^{-}\right)\right| .\right.
\end{aligned}
$$

Applying Proposition 4.2, (5.5), Corollary 5.8 and using (1.1d), we deduce from the above equation that

$$
\begin{aligned}
& \int_{-1}^{h(t)}\left[\left(\partial_{t} u\right)^{2}+\left(\partial_{x x} u\right)^{2}\right] \mathrm{d} x+\frac{\mathrm{d}}{\mathrm{d} t} \int_{-1}^{h(t)}\left(\partial_{x} u\right)^{2} \mathrm{~d} x \\
& \quad \leqslant C\left[1+\int_{-1}^{h(t)}\left[\left(u \partial_{x} u\right)^{2}+\left(\partial_{x} \rho\right)^{2}\right] \mathrm{d} x+\left(\partial_{x} u\left(t, h(t)^{-}\right)^{2}+\left(\partial_{x} u\left(t, h(t)^{+}\right)^{2}\right] \quad(t \in[0, \tau)),\right.\right.
\end{aligned}
$$

where $C=C(M, \tau)>0$. We now estimate $\left\|\partial_{x} u(t, \cdot)\right\|_{L^{2}[-1, h(t)]}$. To this aim we first note that

$$
\left(\partial_{x} u\right)^{2}(t, x)=\left(\partial_{x} u\right)^{2}(t, y)+2 \int_{x}^{y}\left(\partial_{x} u\right)(t, z)\left(\partial_{x x} u\right)(t, z) \mathrm{d} z \quad(t \in[0, \tau), \quad x, y \in[-1, h(t)]) .
$$

Integrating the above relation with respect to $y$ over $[-1, h(t)]$ we get

$$
(1+h(t))\left(\partial_{x} u\right)^{2}(t, x) \leqslant \int_{-1}^{h(t)}\left(\partial_{x} u\right)^{2}(t, y) \mathrm{d} y+2(1+h(t)) \int_{-1}^{h(t)}\left|\partial_{x} u(t, y) \partial_{x x} u(t, y)\right| \mathrm{d} y .
$$

Using (5.15) and Lemma 6.2, we obtain for every $t \in[0, \tau)$

$$
\sup _{-1 \leqslant x<h(t)}\left(\partial_{x} u\right)^{2}(t, x) \leqslant\left(C+\frac{1}{\varepsilon}\right) \int_{-1}^{h(t)}\left(\partial_{x} u\right)^{2}(t, y) \mathrm{d} y+\varepsilon \int_{-1}^{h(t)}\left(\partial_{x x} u\right)^{2}(t, y) \mathrm{d} y .
$$

Combining the above estimate with (6.7) and Lemma 6.1, we obtain that for $\varepsilon$ small enough,

$$
\int_{-1}^{h(t)}\left[\left(\partial_{t} u\right)^{2}+\left(\partial_{x x} u\right)^{2}\right] \mathrm{d} x+\frac{\mathrm{d}}{\mathrm{d} t} \int_{-1}^{h(t)}\left(\partial_{x} u\right)^{2} \mathrm{~d} x \leqslant C\left[1+\int_{-1}^{h(t)}\left[\left(\partial_{x} u\right)^{2}+\left(\partial_{x} \rho\right)^{2}\right] \mathrm{d} x\right] .
$$

Applying Gronwall's lemma, we get

$$
\int_{-1}^{h(t)}\left(\partial_{x} u\right)^{2}(t, x) \mathrm{d} x \leqslant C\left[1+\int_{0}^{t} \int_{-1}^{h(s)}\left(\partial_{x} \rho\right)^{2}(s, x) \mathrm{d} x \mathrm{~d} s\right] \quad(t \in[0, \tau)),
$$


where $C=C(M, \tau)>0$. Moreover, integrating (6.10) with respect to $t$ and using the last formula we obtain that for every $t \in[0, \tau)$ we have

$$
\begin{aligned}
& \int_{0}^{t} \int_{-1}^{h(s)}\left[\left(\partial_{t} u(s, x)\right)^{2}+\left(\partial_{x x} u(s, x)\right)^{2}\right] \mathrm{d} x \mathrm{~d} s+\int_{-1}^{h(t)}\left(\partial_{x} u(t, x)\right)^{2} \mathrm{~d} x \\
& \leqslant C\left[1+\int_{0}^{t} \int_{-1}^{h(s)}\left(\partial_{x} \rho(s, x)\right)^{2} \mathrm{~d} x \mathrm{~d} s+\int_{0}^{t} \int_{0}^{s} \int_{-1}^{h(\sigma)}\left(\partial_{x} \rho\right)^{2}(\sigma, x) \mathrm{d} x \mathrm{~d} \sigma \mathrm{d} s\right] \\
& \leqslant C\left[1+\tau \int_{0}^{t} \int_{-1}^{h(s)}\left(\partial_{x} \rho(s, x)\right)^{2} \mathrm{~d} x \mathrm{~d} s\right],
\end{aligned}
$$

so that we obtain the first inequality in the statement.

The proof of the second inequality is completely similar so we skip it.

We are now in a position to prove our main result.

Proof of Theorem 1.1. Assume for a moment that we have shown the existence of a constant $C=C(M, \tau)>0$ such that

$$
\sup _{t \in[0, \tau)} \int_{-1}^{h(t)}\left(\partial_{x} \rho\right)^{2}(t, x) \mathrm{d} x \leqslant C, \quad \sup _{t \in[0, \tau)} \int_{h(t)}^{1}\left(\partial_{x} \rho\right)^{2}(t, x) \mathrm{d} x \leqslant C .
$$

By combining the above estimate with Corollary 5.8, Lemma 6.3 and (5.15) it follows that there exists a constant $C \geqslant 1$, depending only on $M$ and $\tau$ such that

$$
\begin{gathered}
\left\|\left.u(t, \cdot)\right|_{H^{1}(-1,1)}+\right\| \rho(t, \cdot)\left\|_{H^{1}\left(-1, h_{0}\right)}+\right\| \rho(t, \cdot) \|_{H^{1}\left(h_{0}, 1\right)} \leqslant C \quad(t \in[0, \tau)), \\
\frac{1}{C} \leqslant \rho(t, x) \leqslant C \quad(t \in[0, \tau), \quad x \in[-1,1] \backslash\{h(t)\}), \\
-1+\frac{1}{C} \leqslant h(t) \leqslant 1-\frac{1}{C} \quad(t \in[0, \tau)) .
\end{gathered}
$$

We can thus apply Theorem 3.1 to conclude that there exists $\delta>0$, depending only on $M$ and $\tau$, such that the considered local in time solution is defined on $[t, t+\delta]$, for every $t \in[0, \tau)$. Thus, given $\tau \in[0, T]$, a local strong solution defined on $[0, \tau)$ cannot be a maximal one. Consequently, the maximal solution is defined on $[0, T]$. Since $T>0$ is arbitrary, we have thus obtained the global existence of strong solutions.

We still have to prove (6.11).

To accomplish this goal, we first note that from (1.1a), we obtain

$$
\partial_{t} \ln (\rho)+u \partial_{x} \ln (\rho)=-\partial_{x} u
$$

and thus

$$
\partial_{t}\left(\frac{\partial_{x} \rho}{\rho}\right)+\partial_{x}\left(u \frac{\partial_{x} \rho}{\rho}\right)=-\partial_{x x} u .
$$

Combining the above equation with (1.1a), we deduce

$$
\rho \partial_{t}\left(\frac{\partial_{x} \rho}{\rho^{2}}\right)+\rho u \partial_{x}\left(\frac{\partial_{x} \rho}{\rho}\right)=-\partial_{x x} u .
$$

Adding the above relation and (1.1b), we obtain

$$
\rho\left[\partial_{t}\left(u+\frac{\partial_{x} \rho}{\rho^{2}}\right]+u \partial_{x}\left(u+\frac{\partial_{x} \rho}{\rho}\right)\right)+\partial_{x}\left(\rho^{\gamma}\right)=0 .
$$

We multiply the above equation by $u+\partial_{x} \rho / \rho^{2}$ and integrate with respect to $x$ over $(-1, h(t))$. By using (1.1a) and $(1.1 \mathrm{c})$, we deduce that for every $t \in[0, \tau)$ we have

$$
\frac{1}{2} \frac{\mathrm{d}}{\mathrm{d} t} \int_{-1}^{h(t)} \rho\left(u+\frac{\partial_{x} \rho}{\rho^{2}}\right)^{2} \mathrm{~d} x-\left[\rho u\left(u+\frac{\partial_{x} \rho}{\rho^{2}}\right)^{2}\right](t,-1)+\gamma \int_{-1}^{h(t)} \rho^{\gamma-1} \partial_{x} \rho\left(u+\frac{\partial_{x} \rho}{\rho^{2}}\right) \mathrm{d} x=0 .
$$


From (1.5), together with (3.2) and (3.3), there exists a constant $C=C(M)$ such that

$$
\left[\rho u\left(u+\frac{\partial_{x} \rho}{\rho^{2}}\right)^{2}\right](t,-1) \leqslant C\left(1+\left(\partial_{x} \rho\right)^{2}(t,-1)\right) \quad(t \in[0, \tau)) .
$$

Using (1.1a) at $x=-1,(3.2)$ and (3.3), we obtain

$$
\left[\rho u\left(u+\frac{\partial_{x} \rho}{\rho^{2}}\right)^{2}\right](t,-1) \leqslant C\left(1+\dot{\rho}_{-1}^{2}(t)+\left(\partial_{x} u\right)^{2}(t,-1)\right) \quad(t \in[0, \tau)) .
$$

Combining the last formula and (6.13) we obtain that for every $t \in[0, \tau)$ we have

$$
\begin{array}{rl}
\frac{1}{2} \frac{\mathrm{d}}{\mathrm{d} t} \int_{-1}^{h(t)} \rho\left(u+\frac{\partial_{x} \rho}{\rho^{2}}\right)^{2} \mathrm{~d} x+\frac{\gamma}{2} \int_{-1}^{h(t)} \rho^{\gamma-3}\left(\partial_{x} \rho\right)^{2} & \mathrm{~d} x \\
& \leqslant C \int_{-1}^{h(t)} \rho^{\gamma+1} u^{2} \mathrm{~d} x+C\left(1+\dot{\rho}_{-1}^{2}(t)+\left(\partial_{x} u\right)^{2}(t,-1)\right)
\end{array}
$$

where $C=C(M)$. Combining the above estimate, Proposition 4.2, Corollary 5.5 and (6.9), we deduce that there exists a positive constant $C=C(M, \tau)$ such that

$$
\begin{aligned}
\frac{\mathrm{d}}{\mathrm{d} t} \int_{-1}^{h(t)} & \rho\left(u+\frac{\partial_{x} \rho}{\rho^{2}}\right)^{2} \mathrm{~d} x+\int_{-1}^{h(t)} \rho^{\gamma-3}\left(\partial_{x} \rho\right)^{2} \mathrm{~d} x \\
\leqslant & \quad C\left(1+\frac{1+\varepsilon}{\varepsilon} \int_{-1}^{h(t)}\left(\partial_{x} u\right)^{2}(t, y) \mathrm{d} y\right)+\dot{\rho}_{-1}^{2}(t)+\varepsilon \int_{-1}^{h(t)}\left(\partial_{x x} u\right)^{2}(t, y) \mathrm{d} y \quad(t \in[0, \tau))
\end{aligned}
$$

for any $\varepsilon>0$. Integrating (6.14) we obtain

$$
\begin{array}{rl}
\int_{-1}^{h(t)} \rho\left(u+\frac{\partial_{x} \rho}{\rho^{2}}\right)^{2} & \mathrm{~d} x+\int_{0}^{t} \int_{-1}^{h(s)} \rho^{\gamma-3}\left(\partial_{x} \rho\right)^{2} \mathrm{~d} x \mathrm{~d} s \\
\leqslant \int_{-1}^{h_{0}} \rho_{0}\left(u_{0}+\frac{\partial_{x} \rho_{0}}{\rho_{0}^{2}}\right)^{2} \mathrm{~d} x+C \int_{0}^{t}\left(1+\int_{-1}^{h(s)}\left(\partial_{x} u\right)^{2}(s, y) \mathrm{d} y\right) \mathrm{d} s & \quad+\int_{0}^{t} \dot{\rho}_{-1}^{2}(s) \mathrm{d} s+\int_{0}^{t} \int_{-1}^{h(s)}\left(\partial_{x x} u\right)^{2}(s, y) \mathrm{d} y \mathrm{~d} s \quad(t \in[0, \tau))
\end{array}
$$

Combining the above estimate with Lemma 6.3 and (H4) yields

$$
\int_{-1}^{h(t)} \rho\left(u+\frac{\partial_{x} \rho}{\rho^{2}}\right)^{2} \mathrm{~d} x \leqslant C\left(1+\int_{0}^{t} \int_{-1}^{h(s)}\left(\partial_{x} \rho\right)^{2} \mathrm{~d} x \mathrm{~d} s\right) \quad(t \in[0, \tau)) .
$$

The above estimate and Proposition 4.2 yield

$$
\int_{-1}^{h(t)} \frac{\left(\partial_{x} \rho\right)^{2}}{\rho^{3}} \mathrm{~d} x \leqslant C\left(1+\int_{0}^{t} \int_{-1}^{h(s)}\left(\partial_{x} \rho\right)^{2} \mathrm{~d} x \mathrm{~d} s\right) \quad(t \in[0, \tau)),
$$

where $C=C(M, \tau)>0$. Combining the above estimate with Corollary 5.8 and with Gronwall's lemma, we finally deduce the the first inequality in (6.11). The second one can be deduced in a completely similar manner, so that we end up the proof of our first main result.

\section{Appendix A. Some BaCkground on the linear transport Equation}

In this appendix we justify, with no claim of originality, some of the results on the linear transport equation which have been used in Section 3. This results are known but we prefer, namely in the less studied case of non homogeneous boundary conditions, to provide precise statements and a short proof.

Given $T>0$ and the functions $w, g:[0, T] \times[0,1] \rightarrow \mathbb{R}$, we consider the equation

$$
\partial_{t} \sigma(t, y)+w(t, y) \partial_{y} \sigma(t, y)=g(t, y) \quad(t \in[0, T], y \in(0,1)),
$$


and we impose the initial condition

$$
\sigma(0, y)=\sigma_{0}(y) \quad(y \in(0,1))
$$

In the case in which $w$ vanishes for $y \in\{0,1\}$ we have the following well-known result:

Proposition A.1. Let $T>0$ and

$$
w \in L^{2}\left([0, T] ; H^{2}(0,1)\right) \cap H^{1}\left((0, T) ; L^{2}[0,1]\right),
$$

be such that $w(t, 0)=w(t, 1)=0$ for every $t \in[0, T]$. Then for every $\sigma_{0} \in H^{1}(0,1)$ and

$$
g \in L^{2}\left([0, T] ; H^{1}(0,1)\right) \cap C\left([0, T] ; L^{2}[0,1]\right),
$$

the system (A.1), (A.2) admits an unique solution

$$
\sigma \in C\left([0, T] ; H^{1}(0,1)\right) \cap C^{1}\left([0, T] ; L^{2}[0,1]\right) .
$$

Moreover, there exists an universal constant $M_{0}$ such that the inequality

$$
\begin{gathered}
\|\sigma\|_{C\left([0, T] ; H^{1}(0,1)\right)} \leqslant M_{0} \exp \left(M_{0}\left(T+\|w\|_{L^{1}\left([0, T] ; H^{2}(0,1)\right)}\right)\right)\left(\left\|\sigma_{0}\right\|_{H^{1}(0,1)}+\|g\|_{L^{2}\left(0, T ; H^{1}(0,1)\right.}\right) \\
\left\|\partial_{t} \sigma\right\|_{C\left([0, T] ; L^{2}(0,1)\right)} \leqslant M_{0}\|w\|_{C\left([0, T] ; H^{1}(0,1)\right)} \exp \left(M_{0}\left(T+\|w\|_{L^{1}\left([0, T] ; H^{2}(0,1)\right)}\right)\right) \\
\left(\left\|\sigma_{0}\right\|_{H^{1}(0,1)}+\|g\|_{L^{2}\left(0, T ; H^{1}(0,1)\right.}\right)+\|g\|_{C\left([0, T] ; L^{2}(0,1)\right)}
\end{gathered}
$$

holds for every $\sigma_{0} \in H^{1}(0,1)$ and $g \in L^{2}\left([0, T] ; H^{1}(0,1)\right) \cap C\left([0, T] ; L^{2}[0,1]\right)$.

For a proof of this result we refer, for instance, to Beirão da Veiga [2, Theorem 2.2] (see also Massey [15]).

In the case in which the value of $w$ for $y=0$ is bounded from below by a strictly positive constant, equations (A.1), (A.2), supplemented with a boundary condition at $y=0$ for $\sigma$, still determine a well-posed system, whose solution satisfies an estimate similar to (A.5). (see, for instance, Rauch and Massey [16]). For the sake of completeness, we state below and we give a short proof of this result, making explicit the estimates we need.

Theorem A.2. Let $T>0$ and let $w$ be a function satisfying (A.3). Assume that

$$
w(t, 0)=w_{L}(t), \quad w(t, 1)=0 \quad(t \in[0, T]),
$$

where $w_{L} \in H^{1}(0, T)$ and $w_{L}(t) \geqslant m_{1}>0$, for all $t \in[0, T]$. Let $\sigma_{L} \in H^{1}(0, T)$ be a strictly positive function. Then for every $\sigma_{0} \in H^{1}(0,1)$, satisfying the compatibility condition $\sigma_{L}(0)=\sigma_{0}(0)$ and $g$ satisfying (A.4), the initial and boundary value formed by (A.1), (A.2) and the boundary condition

$$
\sigma(t, 0)=\sigma_{L}(t) \quad(t \in[0, T])
$$

admits a unique solution

$$
\sigma \in C\left([0, T] ; H^{1}(0,1)\right) \cap C^{1}\left([0, T] ; L^{2}[0,1]\right)
$$

Moreover, there exists a constant $M_{0}$, depending on $m_{1}$ such that the inequalities

$$
\begin{gathered}
\|\sigma\|_{C\left([0, T] ; H^{1}(0,1)\right)} \leqslant M_{0} \exp \left(M_{0}\left(T+\|w\|_{L^{1}\left([0, T] ; H^{2}(0,1)\right)}\right)\right)\left(\left\|\sigma_{0}\right\|_{H^{1}(0,1)}+\left\|w_{L}\right\|_{L^{\infty}[0, T]}^{1 / 2}\left\|\sigma_{L}\right\|_{H^{1}(0, T)}\right. \\
\left.+\|g\|_{L^{2}\left([0, T] ; H^{1}(0,1)\right)}\right) \\
\left\|\partial_{t} \sigma\right\|_{C\left([0, T] ; L^{2}[0,1]\right)} \leqslant M_{0}\|w\|_{C\left([0, T] ; H^{1}(0,1)\right)} \exp \left(M_{0}\left(T+\|w\|_{L^{1}\left([0, T] ; H^{2}(0,1)\right)}\right)\right) \\
\times\left(\left\|\sigma_{0}\right\|_{H^{1}(0,1)}+\left\|w_{L}\right\|_{L^{\infty}[0, T]}^{1 / 2}\left\|\sigma_{L}\right\|_{H^{1}(0, T)}+\|g\|_{L^{2}\left([0, T] ; H^{1}(0,1)\right)}\right)+\|g\|_{C\left([0, T] ; L^{2}[0,1]\right)}
\end{gathered}
$$

holds for every $\sigma_{0} \in H^{1}(0,1)$ and $g$ satisfying (A.4). 
Proof of Proposition A.2. To obtain (A.9) we multiply (A.1) by $\sigma$ and we integrate by parts. We obtain in this way that

$$
\frac{\mathrm{d}}{\mathrm{d} t} \int_{0}^{1} \sigma^{2}(t, y) \mathrm{d} y-\int_{0}^{1}\left(\partial_{y} w\right) \sigma^{2}(t, y) \mathrm{d} y-w_{L}(t) \sigma_{L}^{2}(t)=2 \int_{0}^{1} g(t, y) \sigma(t, y) \mathrm{d} y \quad(t \in(0, T)) .
$$

The above relation and Sobolev embeddings yield

$$
\frac{\mathrm{d}}{\mathrm{d} t}\|\sigma(t, \cdot)\|_{L^{2}[0,1]}^{2} \leqslant C\left(1+\|w(t, \cdot)\|_{H^{2}(0,1)}\right)\|\sigma(t, \cdot)\|_{L^{2}[0,1]}^{2}+w_{L}(t) \sigma_{L}^{2}(t)+\|g(t, \cdot)\|_{L^{2}[0,1]}^{2} \quad(t \in(0, T)) .
$$

We then differentiate (A.1) with respect to $y$, multiply by $\partial_{y} \sigma$ and integrate by parts, to get, for all $t \in(0, T)$ :

$$
\frac{\mathrm{d}}{\mathrm{d} t} \int_{0}^{1}\left(\partial_{y} \sigma\right)^{2}(t, y) \mathrm{d} y+\int_{0}^{1}\left[\left(\partial_{y} w\right)\left(\partial_{y} \sigma\right)^{2}\right](t, y) \mathrm{d} y-w_{L}(t)\left(\partial_{y} \sigma(t, 0)\right)^{2}=2 \int_{0}^{1}\left(\partial_{y} g\right)(t, y)\left(\partial_{y} \sigma\right)(t, y) \mathrm{d} y
$$

On the other hand, from (A.1) we obtain

$$
\dot{\sigma}_{L}(t)+\partial_{y} \sigma(t, 0) w_{L}(t)=g(t, 0) \quad(t \in(0, T)) .
$$

This yields

$$
\left(\partial_{y} \sigma(t, 0)\right)^{2} \leqslant 2 \frac{g^{2}(t, 0)}{w_{L}^{2}(t)}+2 \frac{\dot{\sigma}_{L}^{2}(t)}{w_{L}^{2}(t)} \leqslant \frac{2}{m_{1}^{2}}\left(g^{2}(t, 0)+\dot{\sigma}_{L}^{2}(t)\right) \quad(t \in(0, T)) .
$$

Using the fact that $w_{L}$ is bounded away from zero, together with (A.12) and (A.13), we deduce that

$$
\frac{\mathrm{d}}{\mathrm{d} t}\left\|\partial_{y} \sigma(t, \cdot)\right\|_{L^{2}[0,1]}^{2} \leqslant C\left[\left(1+\|w(t, \cdot)\|_{H^{2}(0,1)}\right)\left\|\partial_{y} \sigma(t, \cdot)\right\|_{L^{2}[0,1]}^{2}+\|g(t, \cdot)\|_{H^{1}[0,1]}^{2}+w_{L}(t) \dot{\sigma}_{L}(t)^{2}\right] .
$$

Summing up (A.11) and (A.14) we obtain

$$
\begin{aligned}
\frac{\mathrm{d}}{\mathrm{d} t}\|\sigma(t, \cdot)\|_{H^{1}(0,1)}^{2} \leqslant C\left(1+\|w(t, \cdot)\|_{H^{2}(0,1)}\right) & \|\sigma(t, \cdot)\|_{H^{1}(0,1)}^{2} \\
& +C\left(w_{L}(t)\left(\sigma_{L}^{2}(t)+\dot{\sigma}_{L}^{2}(t)\right)+\|g(t, \cdot)\|_{H^{1}(0,1)}^{2}\right) \quad(t \in(0, T))
\end{aligned}
$$

Applying Gronwall's lemma we deduce (A.9).

Finally, (A.10) is obtained by combining the estimate (A.9) and (A.1).

\section{REFERENCES}

[1] S. S. Antman and J. P. Wilber, The asymptotic problem for the springlike motion of a heavy piston in a viscous gas, Quart. Appl. Math., 65 (2007), pp. 471-498.

[2] H. Beirão DA Veiga, Boundary-value problems for a class of first order partial differential equations in Sobolev spaces and applications to the Euler flow, Rend. Sem. Mat. Univ. Padova, 79 (1988), pp. 247-273.

[3] S. Y. Belov, On the initial-boundary value problems for barotropic motions of a viscous gas in a region with permeable boundaries, J. Math. Kyoto Univ., 34 (1994), pp. 369-389.

[4] M. Boulakia and S. Guerrero, A regularity result for a solid-fluid system associated to the compressible Navier-Stokes equations, Ann. Inst. H. Poincaré Anal. Non Linéaire, 26 (2009), pp. 777-813.

[5] R. M. Brooks and K. Schmitt, The contraction mapping principle and some applications, vol. 9 of Electronic Journal of Differential Equations. Monograph, Texas State University-San Marcos, Department of Mathematics, San Marcos, TX, 2009.

[6] N. Cîndea, S. Micu, I. Rovenţa, and M. Tucsnak, Particle supported control of a fluid-particle system, J. Math. Pures Appl. (9), 104 (2015), pp. 311-353.

[7] B. Desjardins and M. J. Esteban, On weak solutions for fluid-rigid structure interaction: compressible and incompressible models, Comm. Partial Differential Equations, 25 (2000), pp. 1399-1413.

[8] S. Ervedoza, O. Glass, and S. Guerrero, Local exact controllability for the 2 and 3-d compressible Navier-Stokes equations. working paper or preprint, Dec. 2015.

[9] S. Ervedoza, O. Glass, S. Guerrero, and J.-P. Puel, Local exact controllability for the one-dimensional compressible Navier-Stokes equation, Arch. Ration. Mech. Anal., 206 (2012), pp. 189-238.

[10] E. FeireisL, On the motion of rigid bodies in a viscous compressible fluid, Arch. Ration. Mech. Anal., 167 (2003), pp. 281-308.

[11] M. Hieber And M. Murata, The $L^{p}$-approach to the fluid-rigid body interaction problem for compressible fluids, Evol. Equ. Control Theory, 4 (2015), pp. 69-87. 
[12] I. A. Kaliev And M. S. Podkuiko, Nonhomogeneous boundary value problems for equations of viscous heat-conducting gas in time-decreasing non-rectangular domains, J. Math. Fluid Mech., 10 (2008), pp. 176-202.

[13] Y. Liu, T. TAKAhashi, And M. TuCSNAK, Single input controllability of a simplified fluid-structure interaction model, ESAIM Control Optim. Calc. Var., 19 (2013), pp. 20-42.

[14] D. MAITY, Some controllability results for linearized compressible Navier-Stokes system, ESAIM Control Optim. Calc. Var., 21 (2015), pp. 1002-1028.

[15] F. J. MASSEY, III, Abstract evolution equations and the mixed problem for symmetric hyperbolic systems, Trans. Amer. Math. Soc., 168 (1972), pp. 165-188.

[16] J. B. Rauch And F. J. Massey, III, Differentiability of solutions to hyperbolic initial-boundary value problems, Trans. Amer. Math. Soc., 189 (1974), pp. 303-318.

[17] V. V. ŠELUhin, Stabilization of the solution of a model problem on the motion of a piston in a viscous gas, Dinamika Sploshn. Sredy, (1978), pp. 134-146, 173.

[18] V. V. Shelukhin, Unique solvability of the problem of the motion of a piston in a viscous gas, Dinamika Sploshn. Sredy, (1977), pp. 132-150.

[19] Motion with a contact discontinuity in a viscous heat conducting gas, Dinamika Sploshn. Sredy, (1982), pp. 131-152.

[20] V. A. VAĬGANT, Nonhomogeneous boundary value problems for equations of a viscous heat-conducting gas, Dinamika Sploshn. Sredy, (1990), pp. 3-21, 212.

[21] J. L. VÁzquez AND E. ZuAzUA, Large time behavior for a simplified 1 D model of fluid-solid interaction, Comm. Partial Differential Equations, 28 (2003), pp. 1705-1738.

[22] — Lack of collision in a simplified $1 D$ model for fluid-solid interaction, Math. Models Methods Appl. Sci., 16 (2006), pp. $637-678$.

Centre for Applicable Mathematics, TifR, Post Bag No. 6503, GKVK Post Office, Bangalore-560065,India

E-mail address: debayan@math.tifrbng.res.in

INRIA NAncy - Grand Est, 615, Rue du Jardin Botanique. 54600 Villers-lès-Nancy. France

E-mail address: takeo.takahashi@inria.fr

Institut de Mathématiques, Université de Bordeaux, Bordeaux INP, CNRS F-33400 Talence, France

E-mail address: marius.tucsnak@u-bordeaux.fr 